\title{
MODULE CATEGORIES OVER FINITE POINTED TENSOR CATEGORIES
}

\author{
CÉSAR GALINDO AND MARTÍN MOMBELLI
}

\begin{abstract}
We study exact module categories over the representation categories of finite-dimensional quasi-Hopf algebras. As a consequence we classify exact module categories over some families of pointed tensor categories with cyclic group of invertible objets of order $p$, where $p$ is a prime number.
\end{abstract}

Mathematics Subject Classification (2010): 18D10, 16W30, 19D23.

Keywords: Tensor category, module category, quasi-Hopf algebra.

\section{INTRODUCTION}

For a given tensor category $\mathcal{C}$ a module category over $\mathcal{C}$, or a $\mathcal{C}$-module, is the categorification of the notion of module over a ring, it consist of an Abelian category $\mathcal{M}$ together with a biexact functor $\otimes: \mathcal{C} \times \mathcal{M} \rightarrow \mathcal{M}$ satisfying natural associativity and unit axioms. A module category $\mathcal{M}$ is exact [EO1] if for any projective object $P \in \mathcal{C}$ and any $M \in \mathcal{M}$ the object $P \otimes M$ is again projective.

The notion of module category has been used with profit in the theory of tensor categories, see [DGNO], ENO1], ENO2]. Interestingly, the notion of module categories is related with diverse areas of mathematics and mathematical physics such as subfactor theory [Oc], [BEK]; extensions of vertex algebras [KO], Calabi-Yau algebras [Gi], Hopf algebras [N], affine Hecke algebras [BO] and conformal field theory, see for example [BFS], CS1], CS2], [FS1], [FS2], O1].

The classification of exact module categories over a given tensor category was undertaken by several authors:

1. When $\mathcal{C}$ is the semisimple quotient of $U_{q}\left(\mathfrak{s l}_{2}\right)$ [Oc], [KO], [EO2],

2. over the category of finite-dimensional $S L_{q}(2)$-comodules [03],

3 . over the tensor categories of representations of finite supergroups EO1,

4. for any group-theoretical tensor category $\mathrm{O} 2$,

5. over the Tambara-Yamagami categories [Ga2, [MM],

Date: March 30, 2018.

The work of M.M. was supported by CONICET, Secyt (UNC), Mincyt (Córdoba) Argentina. 
6. over the Hageerup fusion categories [GS],

7. over $\operatorname{Rep}(H)$, where $H$ is a lifting of a quantum linear space Mo2.

In this paper we are concerned with the classification of exact module categories over some families of finite non-semisimple pointed tensor categories that are not equivalent to the representation categories of Hopf algebras.

An object $X$ in a tensor category is invertible if there is another object $Y$ such that $X \otimes Y \simeq \mathbf{1} \simeq Y \otimes X$. A pointed tensor category is a tensor category such that every simple object is invertible. The invertible objects form a group. Pointed tensor categories with cyclic group of invertible objects were studied in [EG1, EG2, EG3] and later in [A].

Any finite pointed tensor category is equivalent to the representation category of a finite-dimensional quasi-Hopf algebra $A$. In the case when the group of invertible elements is a cyclic group $G$ there exists an action of $G$ on $\operatorname{Rep}(A)$ such that the equivariantization $\operatorname{Rep}(A)^{G}$ is equivalent to the representation category of a finite-dimensional pointed Hopf algebra $H$, see [A]. The purpose of this work is to relate module categories over $\operatorname{Rep}(A)$ and module categories over $\operatorname{Rep}(H)$ and whenever is possible obtain a classification of exact module categories over $\operatorname{Rep}(A)$ assuming that we know the classification for $\operatorname{Rep}(H)$. Module categories over any quasi-Hopf algebra are parameterized by Morita equivariant equivalence classes of comodule algebras. We would like to establish a correspondence as follows:

$$
\left\{\begin{array}{c}
\text { Morita quivalence classes } \\
\text { of } H \text {-comodule algebras } \\
\text { such that } G \subseteq K_{0}
\end{array}\right\} \longleftrightarrow\left\{\begin{array}{c}
\text { Morita equivalence classes } \\
\text { of } A \text {-comodule algebras } \\
\left(\mathcal{K}, \Phi_{\lambda}\right)
\end{array}\right\} \text {. }
$$

The contents of the paper are the following. In Section 2 we recall the notion of exact module category, the notion of tensor product of module categories over a tensor category. In Section 3 we recall the notion of $G$ graded tensor categories, $G$-actions of tensor categories and crossed products tensor categories. We also recall the $G$-equivariantization construction of tensor categories and module categories.

Section 4 is devoted to study comodule algebras over quasi-Hopf algebras and how they give rise to module categories. Next, in Section 5 we study the equivariantization of the representation category of a quasi-Hopf algebra and the equivariantization of comodule algebras. We describe the datum that gives rise to an action in a representation category of a comodule algebra, that we call a crossed system and we prove that the equivariantization of module categories are modules over a certain crossed product comodule algebra.

In Section 6.1 we recall the definition of a family of finite-dimensional basic quasi-Hopf algebras introduced by I. Angiono [A that are denoted by $A(H, s)$, where $H$ is a coradically graded Hopf algebra with cyclic group of group-like elements. A particular class of these quasi-Hopf algebras were 
introduced by S. Gelaki Ge and later used by Etingof and Gelaki to classify certain families of pointed tensor categories. There is an action of a group $G \subseteq G(H)$ on $\operatorname{Rep}(A(H, s))$ such that $\operatorname{Rep}(A(H, s))^{G} \simeq \operatorname{Rep}(H)$ A. For any left $H$-comodule algebra $K$ such that $\mathbb{k} G \subseteq K_{0}$ we construct a left $A(H, s)$-comodule algebra. We prove that in the case that $|G(H)|=p^{2}$, where $p$ is a prime number, the representation category of this family of comodule algebras is big enough to contain all module categories over $\operatorname{Rep}(A(H, s))$. We apply this result to classify module categories in the case when $H$ is the bosonization of a quantum linear space.

Acknowledgments. We are very grateful to Iván Angiono for many fruitful conversations and for patiently answering our questions on his work $[\mathrm{A}]$. We also thank the referee for his constructive comments.

\section{Preliminaries And notation}

Hereafter $\mathbb{k}$ will denote an algebraically closed field of characteristic 0 . All vector spaces and algebras will be considered over $\mathbb{k}$.

If $H$ is a Hopf algebra and $A$ is an $H$-comodule algebra via $\lambda: A \rightarrow H \otimes_{\mathbb{k}} A$, we shall say that a (right) ideal $J$ is $H$-costable if $\lambda(J) \subseteq H \otimes_{\mathbb{k}} J$. We shall say that $A$ is (right) $H$-simple, if there is no nontrivial (right) ideal $H$ costable in $A$.

If $H$ is a finite-dimensional Hopf algebra then $H_{0} \subseteq H_{1} \subseteq \cdots \subseteq H_{m}=H$ will denote the coradical filtration. When $H_{0} \subseteq H$ is a Hopf subalgebra then the associated graded algebra gr $H$ is a coradically graded Hopf algebra. If $(A, \lambda)$ is a left $H$-comodule algebra, the coradical filtration on $H$ induces a filtration on $A$, given by $A_{n}=\lambda^{-1}\left(H_{n} \otimes_{\mathbb{k}} A\right)$ called the Loewy filtration.

1.1. Finite tensor categories and tensor functors. A tensor category over $\mathbb{k}$ is a $\mathbb{k}$-linear Abelian rigid monoidal category. A finite tensor category EO1] is a tensor category such that it has a finite number of isomorphism classes of simple objects, Hom spaces are finite-dimensional $\mathbb{k}$-vector spaces, all objects have finite length, every simple object has a projective cover and the unit object is simple.

Hereafter all tensor categories will be considered over $\mathbb{k}$ and every functor will be assumed to be $\mathbb{k}$-linear.

If $\mathcal{C}, \mathcal{D}$ are tensor categories, the collection $(F, \xi, \phi): \mathcal{C} \rightarrow \mathcal{D}$ is a tensor functor if $F: \mathcal{C} \rightarrow \mathcal{D}$ is a functor, $\phi: F\left(\mathbf{1}_{\mathcal{C}}\right) \rightarrow \mathbf{1}_{\mathcal{D}}$ is an isomorphism and for any $X, Y \in \mathcal{C}$ the family of natural isomorphisms $\zeta_{X, Y}: F(X) \otimes F(Y) \rightarrow$ $F(X \otimes Y)$ satisfies

$$
\zeta_{X, Y \otimes Z}\left(\operatorname{id}_{F(X)} \otimes \zeta_{Y, Z}\right) a_{F(X), F(Y), F(Z)}=F\left(a_{X, Y, Z}\right) \zeta_{X \otimes Y, Z}\left(\zeta_{X, Y} \otimes \operatorname{id}_{F(Z)}\right),
$$

$$
\begin{gathered}
l_{F(X)}=F\left(l_{X}\right) \zeta_{\mathbf{1}, X}\left(\phi \otimes \operatorname{id}_{F(X)}\right), \\
r_{F(X)}=F\left(r_{X}\right) \zeta_{X, \mathbf{1}}\left(\operatorname{id}_{F(X)} \otimes \phi\right),
\end{gathered}
$$


If $(F, \zeta),(G, \xi): \mathcal{C} \rightarrow \mathcal{D}$ are tensor functors, a natural tensor transformation $\gamma: F \rightarrow G$ is a natural transformation such that $\gamma_{X \otimes Y} \zeta_{X, Y}=$ $\xi_{X, Y}\left(\gamma_{X} \otimes \gamma_{Y}\right)$ for all $X, Y \in \mathcal{C}$.

\section{Module Categories}

A (left) module category over a tensor category $\mathcal{C}$ is an Abelian category $\mathcal{M}$ equipped with an exact bifunctor $\bar{\otimes}: \mathcal{C} \times \mathcal{M} \rightarrow \mathcal{M}$, that we will sometimes refer as the action, natural associativity and unit isomorphisms $m_{X, Y, M}:(X \otimes Y) \bar{\otimes} M \rightarrow X \otimes(Y \bar{\otimes} M), \ell_{M}: \mathbf{1} \bar{\otimes} M \rightarrow M$ subject to natural associativity and unity axioms. See for example [EO1]. A module category $\mathcal{M}$ is exact, EO1, if for any projective object $P \in \mathcal{C}$ the object $P \bar{\otimes} M$ is projective in $\mathcal{M}$ for all $M \in \mathcal{M}$. Sometimes we shall also say that $\mathcal{M}$ is a $\mathcal{C}$-module. Right module categories and bimodule categories are defined similarly.

If $\mathcal{M}$ is a left $\mathcal{C}$-module then $\mathcal{M}^{\text {op }}$ is the right $\mathcal{C}$-module over the opposite Abelian category with action $\mathcal{M}^{\text {op }} \times \mathcal{C} \rightarrow \mathcal{M}^{\text {op }},(M, X) \mapsto X^{*} \bar{\otimes} M$ and associativity isomorphisms $m_{M, X, Y}^{\mathrm{op}}=m_{Y^{*}, X^{*}, M}$ for all $X, Y \in \mathcal{C}, M \in \mathcal{M}$.

If $\mathcal{C}, \mathcal{C}^{\prime}, \mathcal{E}$ are tensor categories, $\mathcal{M}$ is a $(\mathcal{C}, \mathcal{E})$-bimodule category and $\mathcal{N}$ is an $\left(\mathcal{E}, \mathcal{C}^{\prime}\right)$-bimodule category, we shall denote the tensor product over $\mathcal{E}$ by $\mathcal{M} \otimes_{\mathcal{E}} \mathcal{N}$. This category is a $\left(\mathcal{C}, \mathcal{C}^{\prime}\right)$-bimodule category. For more details on the tensor product of module categories the reader is referred to [ENO3, $\mathrm{Gr}$.

A module functor between module categories $\mathcal{M}$ and $\mathcal{M}^{\prime}$ over a tensor category $\mathcal{C}$ is a pair $(T, c)$, where $T: \mathcal{M} \rightarrow \mathcal{M}^{\prime}$ is a functor and $c_{X, M}$ : $T(X \bar{\otimes} M) \rightarrow X \bar{\otimes} T(M)$ is a natural isomorphism such that for any $X, Y \in \mathcal{C}$, $M \in \mathcal{M}:$

$$
\begin{aligned}
\left(\operatorname{id}_{X} \otimes c_{Y, M}\right) c_{X, Y \bar{\otimes}} T\left(m_{X, Y, M}\right) & =m_{X, Y, T(M)} c_{X \otimes Y, M} \\
\ell_{T(M)} c_{1, M} & =T\left(\ell_{M}\right) .
\end{aligned}
$$

We shall use the notation $(T, c): \mathcal{M} \rightarrow \mathcal{M}^{\prime}$. There is a composition of module functors: if $\mathcal{M}^{\prime \prime}$ is another module category and $(U, d): \mathcal{M}^{\prime} \rightarrow \mathcal{M}^{\prime \prime}$ is another module functor then the composition

$$
(U \circ T, e): \mathcal{M} \rightarrow \mathcal{M}^{\prime \prime}, \quad \text { where } e_{X, M}=d_{X, U(M)} \circ U\left(c_{X, M}\right),
$$

is also a module functor.

Let $\mathcal{M}_{1}$ and $\mathcal{M}_{2}$ be module categories over $\mathcal{C}$. We denote by $\operatorname{Hom}_{\mathcal{C}}\left(\mathcal{M}_{1}, \mathcal{M}_{2}\right)$ the category whose objects are module functors $(\mathcal{F}, c)$ from $\mathcal{M}_{1}$ to $\mathcal{M}_{2}$. A morphism between $(\mathcal{F}, c)$ and $(\mathcal{G}, d) \in \operatorname{Hom}_{\mathcal{C}}\left(\mathcal{M}_{1}, \mathcal{M}_{2}\right)$ is a natural transformation $\alpha: \mathcal{F} \rightarrow \mathcal{G}$ such that for any $X \in \mathcal{C}, M \in \mathcal{M}_{1}$ :

$$
d_{X, M} \alpha_{X \bar{\otimes} M}=\left(\operatorname{id}_{X} \bar{\otimes} \alpha_{M}\right) c_{X, M} .
$$

Two module categories $\mathcal{M}_{1}$ and $\mathcal{M}_{2}$ over $\mathcal{C}$ are equivalent if there exist module functors $F: \mathcal{M}_{1} \rightarrow \mathcal{M}_{2}$ and $G: \mathcal{M}_{2} \rightarrow \mathcal{M}_{1}$ and natural isomorphisms id $\mathcal{M}_{1} \rightarrow F \circ G$, id $\mathcal{M}_{2} \rightarrow G \circ F$ that satisfy (2.4). 
The direct sum of two module categories $\mathcal{M}_{1}$ and $\mathcal{M}_{2}$ over a tensor category $\mathcal{C}$ is the $\mathbb{k}$-linear category $\mathcal{M}_{1} \times \mathcal{M}_{2}$ with coordinate-wise module structure. A module category is indecomposable if it is not equivalent to a direct sum of two non trivial module categories.

If $(F, \xi): \mathcal{C} \rightarrow \mathcal{C}$ is a tensor functor and $(\mathcal{M}, \bar{\otimes}, m)$ is a module category over $\mathcal{C}$ we shall denote by $\mathcal{M}^{F}$ the module category $\left(\mathcal{M}, \bar{\otimes}^{F}, m^{F}\right)$ with the same underlying Abelian category with action and associativity isomorphisms defined by

$$
X \bar{\otimes}^{F} M=F(X) \bar{\otimes} M, \quad m_{X, Y, M}^{F}=m_{F(X), F(Y), M}\left(\xi_{X, Y}^{-1} \bar{\otimes} \mathrm{id}_{M}\right),
$$

for all $X, Y \in \mathcal{C}, M \in \mathcal{M}$.

\section{Equivariantization of tensor Categories}

3.1. Group actions on tensor categories. We briefly recall the group actions on tensor categories and the equivariantization construction. For more details the reader is referred to [DGNO].

Let $\mathcal{C}$ be a tensor category and let $\operatorname{Aut}_{\otimes}(\mathcal{C})$ be the monoidal category of tensor auto-equivalences of $\mathcal{C}$, arrows are tensor natural isomorphisms and tensor product the composition of monoidal functors. We shall denote by $\operatorname{Aut}_{\otimes}(\mathcal{C})$ the group of isomorphisms classes of tensor auto-equivalences of $\mathcal{C}$, with the multiplication induced by the composition, i.e. $[F]\left[F^{\prime}\right]=\left[F \circ F^{\prime}\right]$.

For any group $G$ we shall denote by $\underline{G}$ the monoidal category where objects are elements of $G$ and tensor product is given by the product of $G$. An action of the group $G$ over a $\mathcal{C}$, is a monoidal functor $*: \underline{G} \rightarrow \operatorname{Aut}_{\otimes}(\mathcal{C})$. In another words for any $\sigma \in G$ there is a tensor functor $\left(F_{\sigma}, \zeta_{\sigma}\right): \overline{\mathcal{C} \rightarrow \mathcal{C} \text {, and }}$ for any $\sigma, \tau \in G$, there are natural tensor isomorphisms $\gamma_{\sigma, \tau}: F_{\sigma} \circ F_{\tau} \rightarrow F_{\sigma \tau}$.

3.2. $G$-graded tensor categories. Let $G$ be a group and $\mathcal{C}$ be a tensor category. We shall say that $\mathcal{C}$ is $G$-graded, if there is a decomposition

$$
\mathcal{C}=\oplus_{\sigma \in G} \mathcal{C}_{\sigma}
$$

of $\mathcal{C}$ into a direct sum of full Abelian subcategories, such that for all $\sigma, \tau \in G$, the bifunctor $\otimes \operatorname{maps} \mathcal{C}_{\sigma} \times \mathcal{C}_{\tau}$ to $\mathcal{C}_{\sigma \tau}$. Given a $G$-graded tensor category $\mathcal{C}$, and a subgroup $H \subset G$, we shall denote by $\mathcal{C}_{H}$ the tensor subcategory $\oplus_{h \in H} \mathcal{C}_{h}$.

3.3. $G$-equivariantization of tensor categories. Let $G$ be a group acting on a tensor category $\mathcal{C}$. An equivariant object in $\mathcal{C}$ is a pair $(X, u)$ where $X \in \mathcal{C}$ is an object together with isomorphisms $u_{\sigma}: F_{\sigma}(X) \rightarrow X$ satisfying

$$
u_{\sigma \tau} \circ\left(\gamma_{\sigma, \tau}\right)_{X}=u_{\sigma} \circ F_{\sigma}\left(u_{\tau}\right),
$$

for all $\sigma, \tau \in G$. A $G$-equivariant morphism $\phi:(V, u) \rightarrow\left(W, u^{\prime}\right)$ between $G$-equivariant objects $(V, f)$ and $(W, \sigma)$, is a morphism $\phi: V \rightarrow W$ in $\mathcal{M}$ such that $\phi \circ u_{\sigma}=u_{\sigma}^{\prime} \circ F_{\sigma}(\phi)$ for all $\sigma \in G$. 
The tensor category of equivariant objects is denoted by $\mathcal{C}^{G}$ and it is called the equivariantization of $\mathcal{C}$. The tensor product of $\mathcal{C}^{G}$ is defined by

$$
(V, u) \otimes\left(W, u^{\prime}\right):=(V \otimes W, \tilde{u}),
$$

where $\tilde{u}_{\sigma}=\left(u_{\sigma} \otimes u_{\sigma}^{\prime}\right) \zeta_{\sigma}^{-1}$, for any $\sigma \in G$. The unit object is $\left(1, \mathrm{id}_{1}\right)$.

3.4. Crossed product tensor categories and $G$-invariant module categories. Given an action $*: \underline{G} \rightarrow \operatorname{Aut}_{\otimes}(\mathcal{C})$ of $G$ on $\mathcal{C}$, the $G$-crossed product tensor category, denoted by $\overline{\mathcal{C}} \backslash \overline{\text { is defined }}$ as follows. As an Abelian category $\mathcal{C} \rtimes G=\bigoplus_{\sigma \in G} \mathcal{C}_{\sigma}$, where $\mathcal{C}_{\sigma}=\mathcal{C}$ as an Abelian category, the tensor product is

$$
[X, \sigma] \otimes[Y, \tau]:=\left[X \otimes F_{\sigma}(Y), \sigma \tau\right], \quad X, Y \in \mathcal{C}, \quad \sigma, \tau \in G,
$$

and the unit object is $[1, e]$. See $[\mathrm{Ta}$ for the associativity constraint and a proof of the pentagon identity.

If $\mathcal{C}=\operatorname{Rep}(A)$ is the representation category of a finite-dimensional quasiHopf algebra $A$ then $\mathcal{C} \rtimes G$ is also a representation category of a finitedimensional quasi-Hopf algebra $B$. This is an immediate consequence of [E01, Prop. 2.6] since each simple object $W \in \mathcal{C} \rtimes G$ is isomorphic to $[V, e] \otimes[1, \sigma]$, where $\sigma \in G$ and $V \in \operatorname{Rep}(A)$ is simple. Let $d: K_{0}(\mathcal{C}) \rightarrow \mathbb{Z}$ the Perron-Frobenius dimension, then $d([V, e] \otimes[1, \sigma])=d(V) d([1, \sigma])=$ $d(V) \in \mathbb{Z}$, where $d([1, \sigma])=1$ because $[1, \sigma]$ is multiplicatively invertible.

3.5. Equivariantization of module categories. We shall explain analogous procedures for equivariantization in module categories. Equivariant module categories appeared in ENO2]. We shall use the approach given in Ga1.

Let $G$ be a group and $\mathcal{C}$ be a tensor category equipped with an action of $G$. Let $\mathcal{M}$ be a module category over $\mathcal{C}$. For any $g \in G$ we shall denote by $\mathcal{M}^{\sigma}$ the module category $\mathcal{M}^{F_{\sigma}}$. If $\sigma \in G$, we shall say that an endofunctor $T: \mathcal{M} \rightarrow \mathcal{M}$ is $\sigma$-invariant if it has a module structure $(T, c): \mathcal{M} \rightarrow \mathcal{M}^{\sigma}$.

If $\sigma, \tau \in G$ and $T$ is $\sigma$-invariant and $U$ is $\tau$-invariant then $T \circ U$ is $\sigma \tau$ invariant. Indeed, let us assume that the functors $(T, c): \mathcal{M} \rightarrow \mathcal{M}^{\sigma},(U, d)$ : $\mathcal{M} \rightarrow \mathcal{M}^{\tau}$ are module functors then $(T \circ U, b): \mathcal{M} \rightarrow \mathcal{M}^{\sigma \tau}$ is a module functor, where

$$
b_{X, M}=\left(\left(\gamma_{\sigma, \tau}\right)_{X} \otimes \mathrm{id}\right) c_{F_{\tau}(X), M} T\left(d_{X, M}\right),
$$

for all $X \in \mathcal{C}, M \in \mathcal{M}$.

Definition 3.1. Let $F \subseteq G$ be a subgroup.

1. The monoidal category of $\sigma$-equivariant functors for some $\sigma \in F$ in $\mathcal{M}$ will be denoted by $\operatorname{Aut}_{\mathcal{C}}^{F}(\mathcal{M})$.

3. An $F$-equivariant module category is a module category $\mathcal{M}$ equipped with a monoidal functor $(\Phi, \mu): \underline{F} \rightarrow \operatorname{Aut}_{\mathcal{C}}^{F}(\mathcal{M})$, such that $\Phi(\sigma)$ is a $\sigma$-invariant functor for any $\sigma \in F$. 
In another words, an $F$-equivariant module category is a module category $\mathcal{M}$ endowed with a family of module functors $\left(U_{\sigma}, c^{\sigma}\right): \mathcal{M} \rightarrow \mathcal{M}^{\sigma}$ for any $\sigma \in F$ and a family of natural isomorphisms $\mu_{\sigma, \tau}:\left(U_{\sigma} \circ U_{\tau}, b\right) \rightarrow\left(U_{\sigma \tau}, c^{\sigma \tau}\right)$ $\sigma, \tau \in F$ such that

$$
\begin{gathered}
\left(\mu_{\sigma, \tau \nu}\right)_{M} \circ U_{\sigma}\left(\mu_{\tau, \nu}\right)_{M}=\left(\mu_{\sigma \tau, \nu}\right)_{M} \circ\left(\mu_{\sigma, \tau}\right)_{U_{\nu}(M)}, \\
c_{X, M}^{\sigma \tau} \circ\left(\mu_{\sigma, \tau}\right)_{X \bar{\otimes} M}=\left(\left(\gamma_{\sigma, \tau}\right)_{X} \bar{\otimes}\left(\mu_{\sigma, \tau}\right)_{M}\right) \circ c_{F_{\tau}(X), U_{\tau}(M)}^{\sigma} \circ U_{\sigma}\left(c_{X, M}^{\tau}\right),
\end{gathered}
$$

for all $\sigma, \tau, \nu \in F, X \in \mathcal{C}, M \in \mathcal{M}$. Equation (3.2) follows from (1.1) and (3.3) follows from (2.4).

Example 3.2. $\mathcal{C}$ is a $G$-equivariant module category over itself. For any $g \in G$ set $\left(U_{\sigma}, c^{\sigma}\right)=\left(F_{\sigma}, \theta_{\sigma}\right)$ and $\mu_{\sigma, \tau}=\gamma_{\sigma, \tau}$ for all $\sigma, \tau \in G$.

If $\mathcal{M}$ is an $F$-equivariant module category, an equivariant object (see ENO2, Def. 5.3]) is an object $M \in \mathcal{M}$ together with isomorphisms $\left\{v_{\sigma}\right.$ : $\left.U_{\sigma}(M) \rightarrow M: \sigma \in F\right\}$ such that for all $\sigma, \tau \in F$

$$
v_{\sigma \tau} \circ\left(\mu_{\sigma, \tau}\right)_{M}=v_{\sigma} \circ U_{\sigma}\left(v_{\tau}\right) .
$$

The category of $F$-equivariant objects is denoted by $\mathcal{M}^{F}$. A morphism between two $F$-equivariant objects $(M, v),\left(M^{\prime}, v^{\prime}\right)$ is a morphism $f: M \rightarrow$ $M^{\prime}$ in $\mathcal{M}$ such that $f \circ v_{\sigma}=v_{\sigma}^{\prime} \circ U_{\sigma}(f)$ for all $\sigma \in F$.

Lemma 3.3. The category $\mathcal{M}^{F}$ is a $\mathcal{C}^{G}$-module category.

Proof. If $(X, u) \in \mathcal{C}^{G}$ and $(M, v) \in \mathcal{M}^{F}$ the action is defined by

$$
(X, u) \bar{\otimes}(M, v)=(X \bar{\otimes} M, \widetilde{v}),
$$

where $\widetilde{v}_{\sigma}=\left(u_{\sigma} \otimes v_{\sigma}\right) c_{X, M}^{\sigma}$ for all $\sigma \in F$. The object $(X \bar{\otimes} M, \widetilde{v})$ is equivariant due to equation (3.3). The associativity isomorphisms are the same as in $\mathcal{M}$.

The notion of $F$-equivariant module category is equivalent to the notion of $\mathcal{C} \rtimes F$-module cateory. If $\mathcal{M}$ is an $F$-equivariant $\mathcal{C}$-module category for some subgroup $F$ of $G$, then $\mathcal{M}$ is a $\mathcal{C} \rtimes F$-module with action $\bar{\otimes}: \mathcal{C} \rtimes F \times \mathcal{M} \rightarrow \mathcal{M}$ given by $[X, g] \bar{\otimes} M=X \bar{\otimes} U_{g}(M)$, for all $X \in \mathcal{C}, g \in F$ and $M \in \mathcal{M}$. The associativity isomorphisms are given by

$$
m_{[X, g],[Y, h], M}=\left(\mathrm{id}_{X} \otimes\left(c_{Y, U_{h}(M)}^{g}\right)^{-1}\left(\mathrm{id}_{F_{g}(Y)} \otimes \mu_{g, h}^{-1}(M)\right)\right) m_{X, F_{g}(Y), U_{g h}(M)},
$$

for all $X, Y \in \mathcal{C}, g, h \in F$ and $M \in \mathcal{M}$.

In the next statement we collect several well-known results that are, by now, part of the folklore of the subject.

Proposition 3.4. Let $G$ be a finite group acting over a finite tensor category $\mathcal{C}$. If $F \subset G$ is a subgroup, and $\mathcal{M}$ is an $F$-equivariant $\mathcal{C}$-module category, then:

1. If $\mathcal{M}$ is an exact (indecomposable) $\mathcal{C}$-module category then $\mathcal{M}$ is an exact (respectively indecomposable) $\mathcal{C} \rtimes F$-module category. 
2. $\mathcal{M}^{F}$ is an exact module category if and only if $\mathcal{M}$ is an exact module category.

3. There is an equivalence of $\mathcal{C}^{G}$-module categories

$$
\mathcal{M}^{F} \simeq \operatorname{Hom}_{\mathcal{C} \rtimes F}(\mathcal{C}, \mathcal{M}) \simeq \mathcal{C}^{\mathrm{op}} \otimes_{\mathcal{C} \rtimes F} \mathcal{M} \simeq\left(\mathcal{C} \rtimes G \otimes_{\mathcal{C} \rtimes F} \mathcal{M}\right)^{G} .
$$

4. If $\mathcal{N}$ is an indecomposable (exact) module category over $\mathcal{C}^{G}$ there exists a subgroup $F$ of $G$ and an $F$-equivariant indecomposable (exact) module category $\mathcal{M}$ over $\mathcal{C}$ such that $\mathcal{N} \simeq \mathcal{M}^{F}$.

5. If $\mathcal{M}_{1}, \mathcal{M}_{2}$ are $G$-equivariant $\mathcal{C}$-module categories such that $\mathcal{M}_{1}^{G} \simeq$ $\mathcal{M}_{2}^{G}$ as $\mathcal{C}^{G}$-module categories then $\mathcal{M}_{1} \simeq \mathcal{M}_{2}$ as $\mathcal{C}$-module categories.

Proof. 1. Let $P \in \mathcal{C} \rtimes G$ be a projective object. Thus, there exists a family of projective objects $P_{\sigma} \in \mathcal{C}$ such that $P=\oplus_{\sigma \in G}\left[P_{\sigma}, \sigma\right]$. Let $M \in \mathcal{M}$, then $P \bar{\otimes} M=\bigoplus_{\sigma \in G} P_{\sigma} \bar{\otimes} U_{\sigma}(M)$, and since $\mathcal{M}$ is an exact $\mathcal{C}$-module category $P_{\sigma} \bar{\otimes} U_{\sigma}(M)$ is projective for all $\sigma$, thus $P \bar{\otimes} M$ is projective.

2. Under the correspondence described in [Ta, Thm. 4.1] is enough to show that a $\mathcal{C} \rtimes F$-module category $\mathcal{M}$ is exact if and only if $\mathcal{M}$ is an exact $\mathcal{C}$-module category. The proof follows from part (1) of this proposition.

3. An object $(F, c) \in \operatorname{Hom}_{\mathcal{C} \rtimes F}(\mathcal{C}, \mathcal{M})$ is determined uniquely by an object $M \in \mathcal{M}$ such that $F(X)=X \bar{\otimes} M$ together with an isomorphism $v_{\sigma}=$ $c_{[\mathbf{1}, \sigma], \mathbf{1}}: U_{\sigma}(M) \rightarrow M$. This correspondence establish an equivalence $\mathcal{M}^{F} \simeq$ $\operatorname{Hom}_{\mathcal{C} \rtimes F}(\mathcal{C}, \mathcal{M})$. The equivalence $\operatorname{Hom}_{\mathcal{C} \rtimes F}(\mathcal{C}, \mathcal{M}) \simeq \mathcal{C}^{\text {op }} \bigotimes_{\mathcal{C} \rtimes F} \mathcal{M}$ follows from [Gr, Thm. 3.20].

Since $\mathcal{C} \rtimes G \bigotimes_{\mathcal{C} \rtimes F} \mathcal{M}$ is a $\mathcal{C} \rtimes G$-module then it is a $G$-equivariant $\mathcal{C}$-module category, thus

$$
\left(\mathcal{C} \rtimes G \otimes_{\mathcal{C} \rtimes F} \mathcal{M}\right)^{G} \simeq \mathcal{C}^{\mathrm{op}}{\bigotimes_{\mathcal{C} \rtimes G}}\left(\mathcal{C} \rtimes G \otimes_{\mathcal{C} \rtimes F} \mathcal{M}\right) \simeq \mathcal{C}^{\mathrm{op}} \otimes_{\mathcal{C} \rtimes F} \mathcal{M} \simeq \mathcal{M}^{F}
$$

The first equivalence is [Ta, Thm 4.1].

4. By [EO1, Proposition 3.9] every indecomposable exact tensor category over a finite tensor category is a simple module category in the sense of Ga1], so the result follows by the main result of [Ga1], and the item (1) of this proposition.

5. Since $\mathcal{M}_{1}, \mathcal{M}_{2}$ are $G$-equivariant then they are $\mathcal{C} \rtimes G$-module categories. It follows from [Ta, Thm. 4.1] that this are equivalent $\mathcal{C} \rtimes G$-module categories. This equivalence induces an equivalence of $\mathcal{C}$-module categories (see [Ta, Ex. 2.5]).

It follows from Proposition 3.4 (4) that the equivariantization construction of module categories by a fixed subgroup is injective. Moreover, if the equivariantization of a module category by two subgroups gives the same result then the groups must be conjugate. We shall give the precise statement in the following. First we need a definition and a result from the paper Ga2. 
Definition 3.5. Ga2, Def. 4.3] Let $\mathcal{C}$ be a $G$-graded tensor category. If $(\mathcal{M}, \otimes)$ is a $\mathcal{C}_{e}$-module category, then a $\mathcal{C}$-extension of $\mathcal{M}$ is a $\mathcal{C}$-module category $(\mathcal{M}, \odot)$ such that $(\mathcal{M}, \otimes)$ is obtained by restriction to $\mathcal{C}_{e}$.

Proposition 3.6. Ga2, Prop. 4.6] Let $\mathcal{C}$ be a $G$-graded finite tensor category and let $F, F^{\prime} \subset G$ be subgroups and $(\mathcal{N}, \odot),\left(\mathcal{N}^{\prime}, \odot^{\prime}\right)$ be a $\mathcal{C}_{F}$-extension and a $\mathcal{C}_{F^{\prime}}$-extension of the indecomposable $\mathcal{C}_{e}$-module categories $\mathcal{N}$ and $\mathcal{N}^{\prime}$, respectively. Then $\mathcal{C} \otimes_{\mathcal{C}_{F^{\prime}}} \mathcal{N}^{\prime} \cong \mathcal{C} \otimes_{\mathcal{C}_{F}} \mathcal{N}$ as $\mathcal{C}$-modules if and only if there exists $\sigma \in G$ such that $F=\sigma F^{\prime} \sigma^{-1}$ and $\mathcal{C}_{\sigma F^{\prime}} \bigotimes_{\mathcal{C}_{F^{\prime}}} \mathcal{N}^{\prime} \cong \mathcal{N}$ as $\mathcal{C}_{e}$-module categories.

Theorem 3.7. Let $G$ be a finite group action on a finite tensor category $\mathcal{C}$ and let $F, F^{\prime} \subset G$ be subgroups. Let $\mathcal{N}$ and $\mathcal{N}^{\prime}$ be an $F$-equivariant and an $F^{\prime}$-equivariant module categories respectively, such that $\mathcal{N}$ and $\mathcal{N}^{\prime}$ are indecomposable as $\mathcal{C}$-module categories and $\mathcal{N}^{F} \cong \mathcal{N}^{\prime F^{\prime}}$ as $\mathcal{C}^{G}$-module categories. Then $F$ and $F^{\prime}$ are conjugate subgroups in $G$.

Proof. It follows from Proposition 3.4 (3) that there is an equivalence of $\mathcal{C}^{G}$-modules

$$
\left(\mathcal{C} \rtimes G \otimes_{\mathcal{C} \rtimes F} \mathcal{N}\right)^{G} \simeq\left(\mathcal{C} \rtimes G \bigotimes_{\mathcal{C} \rtimes F^{\prime}} \mathcal{N}^{\prime}\right)^{G} .
$$

Hence by Proposition 3.4 (5) there is an equivalence of $\mathcal{C} \rtimes G$-modules $\mathcal{C} \rtimes$ $G \otimes_{\mathcal{C} \rtimes F} \mathcal{N} \simeq \mathcal{C} \rtimes G \otimes_{\mathcal{C} \rtimes F^{\prime}} \mathcal{N}^{\prime}$, thus the result follows from Proposition 3.6.

\section{Quasi-Hopf ALGEBRAS}

A quasi-bialgebra [D] is a four-tuple $(A, \Delta, \varepsilon, \Phi)$ where $A$ is an associative algebra with unit, $\Phi \in(A \otimes A \otimes A)^{\times}$is called the associator, and $\Delta: A \rightarrow$ $A \otimes A, \varepsilon: A \rightarrow k$ are algebra homomorphisms satisfying the identities

$$
\begin{aligned}
& \Phi(\Delta \otimes \text { id })(\Delta(h))=(\operatorname{id} \otimes \Delta)(\Delta(h)) \Phi, \\
& (\operatorname{id} \otimes \varepsilon)(\Delta(h))=h \otimes 1, \quad(\varepsilon \otimes \text { id })(\Delta(h))=1 \otimes h,
\end{aligned}
$$

for all $h \in A$. The associator $\Phi$ has to be a 3-cocycle, in the sense that

$$
\begin{aligned}
& (1 \otimes \Phi)(\mathrm{id} \otimes \Delta \otimes \text { id })(\Phi)(\Phi \otimes 1)=(\text { id } \otimes \text { id } \otimes \Delta)(\Phi)(\Delta \otimes \text { id } \otimes \text { id })(\Phi), \\
& (\text { id } \otimes \varepsilon \otimes \text { id })(\Phi)=1 \otimes 1 \otimes 1 .
\end{aligned}
$$

$A$ is called a quasi-Hopf algebra if, moreover, there exists an anti-morphism $S$ of the algebra $A$ and elements $\alpha, \beta \in A$ such that, for all $h \in A$, we have:

$$
\begin{aligned}
& S\left(h_{(1)}\right) \alpha h_{(2)}=\varepsilon(h) \alpha \quad \text { and } \quad h_{(1)} \beta S\left(h_{(2)}\right)=\varepsilon(h) \beta, \\
& \Phi^{1} \beta S\left(\Phi^{2}\right) \alpha \Phi^{3}=1 \quad \text { and } S\left(\Phi^{-1}\right) \alpha \Phi^{-2} \beta S\left(\Phi^{-3}\right)=1 .
\end{aligned}
$$

Here we use the notation $\Phi=\Phi^{1} \otimes \Phi^{2} \otimes \Phi^{3}, \Phi^{-1}=\Phi^{-1} \otimes \Phi^{-3} \otimes \Phi^{-3}$. If $A$ is a quasi-Hopf algebra, we shall denote by $\operatorname{Rep}(A)$ the tensor category of finite-dimensional representations of $A$. 
An invertible element $J \in A \otimes A$ is called a twist if $(\varepsilon \otimes$ id $)(J)=1=$ (id $\otimes \varepsilon)(J)$. If $A$ is a quasi-Hopf algebra and $J=J^{1} \otimes J^{2} \in A \otimes A$ is a twist with inverse $J^{-1}=J^{-1} \otimes J^{-2}$, then we can define a quasi-Hopf algebra on the same algebra $A$ keeping the counit and antipode and replacing the comultiplication, associator and the elements $\alpha$ and $\beta$ by

$$
\begin{aligned}
& \Delta_{J}(h)=J \Delta(h) J^{-1}, \\
& \Phi_{J}=(1 \otimes J)(\mathrm{id} \otimes \Delta)(J) \Phi(\Delta \otimes \mathrm{id})\left(J^{-1}\right)\left(J^{-1} \otimes 1\right), \\
& \alpha_{J}=S\left(J^{-1}\right) \alpha J^{-2}, \quad \beta_{J}=J^{1} \beta S\left(J^{2}\right) .
\end{aligned}
$$

We shall denote this new quasi-Hopf algebra by $\left(A_{J}, \Phi_{J}\right)$. If $\Phi=1$ then, in this case, we shall denote $\Phi_{J}=d J$.

4.1. Comodule algebras over quasi-Hopf algebras. Let $(A, \Phi, \alpha, \beta, 1)$ be a finite dimensional quasi-Hopf algebra.

Definition 4.1. A left $A$-comodule algebra is a family $\left(\mathcal{K}, \lambda, \Phi_{\lambda}\right)$ such that $\mathcal{K}$ is an algebra, $\lambda: \mathcal{K} \rightarrow A \otimes \mathcal{K}$ is an algebra map, $\Phi_{\lambda} \in A \otimes A \otimes \mathcal{K}$ is an invertible element such that

$\left(1 \otimes \Phi_{\lambda}\right)(\mathrm{id} \otimes \Delta \otimes \mathrm{id})\left(\Phi_{\lambda}\right)(\Phi \otimes 1)=(\mathrm{id} \otimes \mathrm{id} \otimes \lambda)\left(\Phi_{\lambda}\right)(\Delta \otimes \mathrm{id} \otimes \mathrm{id})\left(\Phi_{\lambda}\right)$,

$$
\begin{gathered}
(\operatorname{id} \otimes \epsilon \otimes \text { id })\left(\Phi_{\lambda}\right)=1, \\
\Phi_{\lambda}(\Delta \otimes \text { id }) \lambda(x)=((\text { id } \otimes \lambda) \lambda(x)) \Phi_{\lambda}, \quad x \in \mathcal{K}
\end{gathered}
$$

We shall say that a comodule algebra $\left(\mathcal{K}, \lambda, \Phi_{\lambda}\right)$ is right $A$-simple if it has no non-trivial right ideals $J \subseteq \mathcal{K}$ such that $J$ is costable, that is $\lambda(J) \subseteq$ $A \otimes \mathcal{K}$.

Remark 4.2. The notion of comodule algebra for quasi-Hopf algebras does not coincide with the notion of comodule algebra for (usual) Hopf algebras. For quasi-Hopf algebras the coaction may not be coassociative.

If $\left(\mathcal{K}, \lambda, \Phi_{\lambda}\right)$ is a left $A$-comodule algebra, the category ${ }_{\mathcal{K}}^{A} \mathcal{M}_{A}$ consists of $(\mathcal{K}, A)$-bimodules $M$ equipped with a $(\mathcal{K}, A)$-bimodule map $\delta: M \rightarrow A \otimes M$ such that for all $m \in M$

$$
\begin{aligned}
\Phi_{\lambda}(\Delta \otimes \mathrm{id}) \delta(m) & =(\mathrm{id} \otimes \delta) \delta(m) \Phi, \\
(\varepsilon \otimes \mathrm{id}) \delta & =\mathrm{id} .
\end{aligned}
$$

The following result will be useful to present examples of exact module categories, it is a consequence of some freeness results on comodule algebras over quasi-Hopf algebras proven by $\mathrm{H}$. Henker.

Lemma 4.3. Let $\left(\mathcal{K}, \lambda, \Phi_{\lambda}\right)$ be a right $A$-simple left $A$-comodule algebra. If $M \in \mathcal{K} \mathcal{M}$ then $A \otimes M \in \mathcal{K}_{\mathcal{M}}$ is projective. 
Proof. The object $A \otimes M$ is in the category ${ }_{\mathcal{K}}^{A} \mathcal{M}_{A}$ as follows. The left $\mathcal{K}$ action and the right $A$-action on $A \otimes M$ are determined by

$$
x \cdot(a \otimes m)=x_{(-1)} a \otimes x_{(0)} \cdot m, \quad(a \otimes m) \cdot b=a b \otimes m,
$$

for all $x \in \mathcal{K}, a, b \in A$ and $m \in M$. The coaction is determined by $\delta$ : $A \otimes M \rightarrow A \otimes A \otimes M, \delta=\Phi_{\lambda}\left(\Delta \otimes \mathrm{id}_{M}\right)$. It follows from [He, Lemma 3.6] that $A \otimes M$ is a projective $\mathcal{K}$-module.

4.2. Comodule algebras over radically graded quasi-Hopf algebras. Let $A$ be a quasi-Hopf algebra radically graded, that is there is an algebra grading $A=\oplus_{i=0}^{m} A[i]$, where $I:=\operatorname{Rad} A=\oplus_{i \geq 1} A[i]$ and $I^{k}=\oplus_{i \geq k} A[i]$ for any $k=0 \ldots m$. Here $I^{0}=A$. Since $\Delta(I) \subseteq I \otimes A+A \otimes I$ then $\Delta(I) \subseteq$ $\sum_{j=0}^{k} I^{j} \otimes I^{k-j}$ for any $k=0 \ldots m$. In this case $A[0]$ is semisimple, $A$ is generated by $A[0]$ and $A[1]$, and the associator $\Phi$ is an element in $A[0]^{\otimes 3}$, see [EG1, Lemma 2.1].

If $\left(\mathcal{K}, \lambda, \Phi_{\lambda}\right)$ is a left $A$-comodule algebra, define

$$
\mathcal{K}_{i}=\lambda^{-1}\left(I^{i} \otimes \mathcal{K}\right), \quad i=0 \ldots m .
$$

This is an algebra filtration, thus we can consider the associated graded algebra gr $\mathcal{K}=\oplus_{i=0}^{m} \mathcal{K}[i], \mathcal{K}[i]=\mathcal{K}_{i} / \mathcal{K}_{i+1}$.

Lemma 4.4. $\quad$ 1. The above filtration satisfies

$$
\lambda\left(\mathcal{K}_{i}\right) \subseteq \sum_{j=0}^{i} I^{j} \otimes \mathcal{K}_{i-j} .
$$

2. There is a left $A$-comodule algebra structure $\left(\operatorname{gr} \mathcal{K}, \bar{\lambda}, \bar{\Phi}_{\lambda}\right)$ satisfying

$$
\bar{\lambda}(\operatorname{gr} \mathcal{K}(n)) \subseteq \oplus_{k=0}^{n} A[k] \otimes \mathcal{K}[n-k] .
$$

3. $\left(\mathcal{K}[0], \bar{\lambda}, \bar{\Phi}_{\lambda}\right)$ is a left $A[0]$-comodule algebra.

Proof. Item (1) follows from the definition of $\mathcal{K}_{i}$ and equation (4.12). For each $n=0 \ldots m$ there is a linear map $\bar{\lambda}: \operatorname{gr} \mathcal{K} \rightarrow A \otimes \operatorname{gr} \mathcal{K}$ such that the following diagram commutes

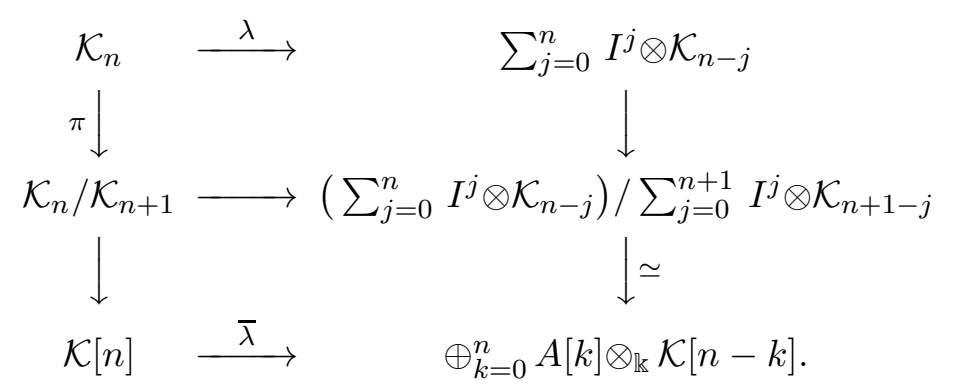

Defining $\bar{\Phi}_{\lambda}$ as the projection of $\Phi_{\lambda}$ to $A[0] \otimes A[0] \otimes \mathcal{K}[0]$ follows immediately that $\left(\operatorname{gr} \mathcal{K}, \bar{\lambda}, \bar{\Phi}_{\lambda}\right)$ is a left $A$-comodule algebra.

Lemma 4.5. The following statements are equivalent: 
1. $\mathcal{K}$ is a right $A$-simple left $A$-comodule algebra.

2. $\mathcal{K}[0]$ is a right $A[0]$-simple left $A[0]$-comodule algebra.

3. gr $\mathcal{K}$ is a right $A$-simple left $A$-comodule algebra.

Proof. Assume $\mathcal{K}[0]$ is a right $A[0]$-simple. Let $J \subseteq A$ be a right ideal $A$-costable. Consider the filtration $J=J_{0} \supseteq J_{1} \supseteq \cdots \supseteq J_{m}$ given by $J_{k}=\lambda^{-1}\left(I^{k} \otimes J\right)$ for all $k=0 \ldots m$. Set $\bar{J}(k)=J_{k} / J_{k+1}$ for any $k$ and $\bar{J}=\oplus_{k} \bar{J}(k)$. It follows that for any $n=0 \ldots m$

$$
\bar{\lambda}(\bar{J}(n)) \subseteq \oplus_{k=0}^{n} A[k] \otimes \bar{J}(k) .
$$

In particular $\bar{J}(0) \subseteq \mathcal{K}[0]$ is a right ideal $A[0]$-costable thus $\bar{J}=\mathcal{K}[0]$ or $\bar{J}=0$. In the first case $J=A$ and in the second case $J=J_{1}$. It follows from (4.17) that $\bar{J}(1) \subseteq \mathcal{K}[0]$ is a a right ideal $A[0]$-costable. Hence $J=J_{2}$. Continuing this reasoning we obtain that $J=0$.

Assume now that $\mathcal{K}$ is a right $A$-simple. Let $\bar{J} \subseteq \mathcal{K}[0]$ be a right $A[0]$ costable ideal. Denote $\pi: \mathcal{K} \rightarrow \mathcal{K}[0]$ the canonical projection and $J=$ $\pi^{-1}(\bar{J})$. Clearly $J$ is a right $A$-costable ideal thus $J=0$ or $J=\mathcal{K}$, thus $\bar{J}=0$ or $\bar{J}=\mathcal{K}[0]$ respectively.

As a consequence we have the following result.

Corollary 4.6. Let $(A, \Phi)$ be a radically graded quasi-Hopf algebra and $\left(\mathcal{K}, \lambda, \Phi_{\lambda}\right)$ be a left $A$-comodule algebra such that $\mathcal{K}[0]=\mathbb{k} 1$ then $A$ is twist equivalent to a Hopf algebra.

Proof. Since $\left(\mathcal{K}[0], \bar{\lambda}, \bar{\Phi}_{\lambda}\right)$ is a left $A[0]$-comodule algebra then there exists an invertible element $J \in A \otimes A$ such that $J \otimes 1=\bar{\Phi}_{\lambda}$. Equation (4.10) implies that $\Phi=d J$.

4.3. Module categories over quasi-Hopf algebras. For any comodule algebra over a quasi-Hopf algebra $A$ there is associated a module category over $\operatorname{Rep}(A)$.

Lemma 4.7. Let $A$ be a finite-dimensional quasi-Hopf algebra.

1. If $\left(\mathcal{K}, \lambda, \Phi_{\lambda}\right)$ is a left $A$-comodule algebra then the category $\mathcal{K} \mathcal{M}$ is a module category over $\operatorname{Rep}(A)$. It is exact if $\mathcal{K}$ is right $A$-simple.

2. If $\mathcal{M}$ is an exact module category over $\operatorname{Rep}(A)$ there exists a left $A$ comodule algebra $\left(\mathcal{K}, \lambda, \Phi_{\lambda}\right)$ such that $\mathcal{M} \simeq \mathcal{K} \mathcal{M}$ as module categories over $\operatorname{Rep}(A)$.

Proof. 1. The action $\bar{\otimes}: \operatorname{Rep}(A) \times \mathcal{K} \mathcal{M} \rightarrow \mathcal{K} \mathcal{M}$ is given by the tensor product over the field $\mathbb{k}$ where the action on the tensor product is given by $\lambda$. The associativity isomorphisms $m_{X, Y, M}:(X \otimes Y) \otimes M \rightarrow X \otimes(Y \otimes M)$ are given by

$$
m_{X, Y, M}(x \otimes y \otimes m)=\Phi_{\lambda}^{1} \cdot x \otimes \Phi_{\lambda}^{2} \cdot y \otimes \Phi_{\lambda}^{3} \cdot m,
$$

for all $x \in X, y \in Y, M \in M, X, Y \in \operatorname{Rep}(A), M \in \mathcal{K} \mathcal{M}$. To prove that $\mathcal{K} \mathcal{M}$ is exact, it is enough to verify that $A \otimes M$ is projective for any $M \in \mathcal{K} \mathcal{M}$ but this is Lemma 4.3. 
2. This is a straightforward consequence of [EO1, Thm. 3.17], the proof of [AM, Prop. 1.19] extends mutatis mutandis to the quasi-Hopf setting.

Definition 4.8. Two left $A$-comodule algebras $\left(\mathcal{K}, \lambda, \Phi_{\lambda}\right),\left(\mathcal{K}^{\prime}, \lambda^{\prime}, \Phi_{\lambda}^{\prime}\right)$ are equivariantly Morita equivalent if the corresponding module categories are equivalent.

4.4. Comodule algebras coming from twisting. Let $(A, \Phi)$ be a quasiHopf algebra and $J \in A \otimes A$ be a twist. Let $\left(K, \lambda, \Phi_{\lambda}\right)$ be a left $A$-comodule algebra. Let us denote by $\left(K_{J}, \lambda_{J}, \widetilde{\Phi}_{\lambda}\right)$ the following left $A_{J}$-comodule algebra. As algebras $K_{J}=K$, the coaction $\lambda_{J}=\lambda$ and $\widetilde{\Phi}_{\lambda}=\Phi_{\lambda}\left(J^{-1} \otimes 1\right)$.

The following results are straightforward.

Lemma 4.9. $\left(K_{J}, \lambda_{J}, \widetilde{\Phi}_{\lambda}\right)$ is a left $A_{J}$-comodule algebra. It is right $A$-simple if and only if $\left(K, \lambda, \Phi_{\lambda}\right)$ is right $A$-simple.

Lemma 4.10. Let $J \in A \otimes A$ be a twist. If $\left(K, \lambda, \Phi_{\lambda}\right)$ and $\left(K^{\prime}, \lambda^{\prime}, \Phi_{\lambda}^{\prime}\right)$ are equivariantly Morita equivalent $A$-comodule algebras then $\left(K_{J}, \lambda_{J}, \Phi_{\lambda}\left(J^{-1} \otimes 1\right)\right)$ and $\left(K_{J}^{\prime}, \lambda_{J}^{\prime}, \Phi_{\lambda}^{\prime}\left(J^{-1} \otimes 1\right)\right)$ are equivariant Morita equivalent $A_{J}$-comodule algebras.

\section{Equivariantization OF QUASI-HopF ALGEBRAS}

For a quasi-Hopf algebra $A$ we shall explain the notion of a crossed system over $A$ and discuss its relation with the equivariantization of the category $\operatorname{Rep}(A)$.

Let $A_{1}, A_{2}$ be quasi-Hopf algebras. A twisted homomorphism between $A_{1}$ and $A_{2}$ is pair $(f, J)$ consisting of a homomorphism of algebras $f: A_{1} \rightarrow A_{2}$ and an invertible element $J \in A_{2}^{\otimes 2}$ such that

$$
\begin{gathered}
\Phi_{2}(\Delta \otimes \mathrm{id})(J)(J \otimes 1)=(\mathrm{id} \otimes \Delta)(J)(1 \otimes J)\left(f^{\otimes 3}\right)\left(\Phi_{1}\right), \\
(\varepsilon \otimes \mathrm{id})(J)=(\mathrm{id} \otimes \varepsilon)(J)=1, \\
\varepsilon(f(a))=\varepsilon(a), \\
\Delta(f(a)) J=J\left(f^{2 \otimes}(\Delta(a))\right), \quad \text { for all } a \in A .
\end{gathered}
$$

Remark 5.1. If $(f, J): A_{1} \rightarrow A_{2}$ is a twisted homomorphism, then $J^{-1} \in$ $A_{2} \otimes A_{2}$ is a twist and $f: A_{1} \rightarrow\left(A_{2}\right)_{J^{-1}}$ is a homomorphism of quasibialgebras.

We define the category $\underline{\text { End }}^{\mathrm{Tw}}\left(A_{1}, A_{2}\right)$ whose objects are twisted homomorphism from $A_{1}$ to $A_{2}$. A morphism between two twisted homomorphisms $(f, J),\left(f^{\prime}, J^{\prime}\right): A_{1} \rightarrow A_{2}$ is an element $c \in A_{2}$ such that $c f(a)=f^{\prime}(a) c$ for any $a \in A_{1}$ and $\Delta(c) J=J^{\prime}(c \otimes c)$. The composition of $a: f \rightarrow g, b: g \rightarrow h$, is $b a: f \rightarrow h$. If $\left(f, J_{f}\right): A_{1} \rightarrow A_{2}$ and $\left(g, J_{g}\right): A_{2} \rightarrow A_{3}$ are twisted homomorphism, we define the composition as the twisted homomorpshism $\left(g \circ f, J_{g}(g \otimes g)\left(J_{f}\right)\right): A_{1} \rightarrow A_{3}$. 
To any twisted homomorphism $(f, J): A_{1} \rightarrow A_{2}$ there is associated a tensor functor

$$
\left(f^{*}, \xi^{J}\right): \operatorname{Rep}\left(A_{2}\right) \rightarrow \operatorname{Rep}\left(A_{1}\right)
$$

where $f^{*}(V)=V$ for all $V \in \operatorname{Rep}\left(A_{2}\right)$, and $f^{*}$ is the identity over arrows. The $A_{1}$-action on $f^{*}(V)$ is given through the morphism $f$. The monoidal structure is given by applying the element $J \in A_{2}^{\otimes 2}$ :

$$
\xi_{M, N}^{J}: f^{*}(M) \otimes f^{*}(N) \rightarrow f^{*}(M \otimes N), \quad \xi_{M, N}^{J}(m \otimes n)=J(m \otimes n),
$$

for any $M, N \in \operatorname{Rep}\left(A_{2}\right), m \in M, n \in N$. Morphisms between twisted homomorphisms $f, f^{\prime}: A_{1} \rightarrow A_{2}$ of quasi-Hopf algebras correspond to tensor natural transformations between the associated tensor functors.

5.1. Crossed system over a quasi-Hopf algebra. Given a quasi-Hopf algebra $A$ we shall denote by $\underline{\operatorname{Aut}}^{\mathrm{Tw}}(A)$ the (monodial) subcategory of $\underline{\text { End }}^{\mathrm{Tw}}(A)$ where objects are twisted automorphisms of $A$, and arrows are isomorphisms of twisted automorphisms.

Let $G$ be a group, and let $A$ be a quasi-Hopf algebra. A $G$-crossed system over $A$ is a monoidal functor $*: \underline{G} \rightarrow \underline{\operatorname{Aut}}^{\mathrm{Tw}}(A)$ such that $e_{*}=\left(\operatorname{id}{ }_{A}, 1 \otimes 1\right)$.

More explicitly a $G$-crossed system consists of the following data:

- A twisted automorphism $\left(\sigma_{*}, J_{\sigma}\right)$ for each $\sigma \in G$,

- an element $\theta_{(\sigma, \tau)} \in A^{\times}$for each $\sigma, \tau \in G$,

such that for all $a \in A, \sigma, \tau, \rho \in G$,

$$
\begin{aligned}
\varepsilon\left(\theta_{(\sigma, \tau)}\right) & =1, \\
\left(1_{*}, J_{1}\right) & =(\mathrm{id}, 1 \otimes 1), \\
\theta_{(\sigma, \tau)}(\sigma \tau)_{*}(a) & =\sigma_{*}\left(\tau_{*}(a)\right) \theta_{(\sigma, \tau)}, \\
\theta_{(\sigma, \tau)} \theta_{(\sigma \tau, \rho)} & =\sigma_{*}\left(\theta_{(\tau, \rho)}\right) \theta_{\sigma, \tau \rho}, \\
\theta_{(1, \sigma)} & =\theta_{(\sigma, 1)}=1, \\
\Delta\left(\theta_{(\sigma, \tau)}\right) J_{\sigma \tau} & =J_{\sigma}\left(\sigma_{*} \otimes \sigma_{*}\right)\left(J_{\tau}\right)\left(\theta_{(\sigma, \tau)} \otimes \theta_{(\sigma, \tau)}\right) .
\end{aligned}
$$

Let $A \# G$ be the vector space $A \otimes_{\mathbb{k}} \mathbb{k} G$ with product and coproduct

$$
(x \# \sigma)(y \# \tau)=x \sigma_{*}(y) \theta_{(\sigma, \tau)} \# \sigma \tau, \quad \Delta(x \# \sigma)=x_{(1)} J_{\sigma}^{1} \# \sigma \otimes x_{(2)} J_{\sigma}^{2} \# \sigma,
$$

for all $x, y \in A, \sigma, \tau \in G$.

Proposition 5.2. The foregoing operations makes the vector space $A \# G$ into a quasi-bialgebra with associator $\Phi^{1} \# e \otimes \Phi^{2} \# e \otimes \Phi^{3} \# e$, and counit $\varepsilon(x \# \sigma)=\varepsilon(x)$ for all $x \in A, \sigma \in G$.

Proof. It is straightforward to see that $A \# G$ is an associative algebra with unit 1\#e. Equation (4.1) follows from (5.1). The map $\varepsilon$ is an algebra morphism by (5.3) and (5.5). Equations (4.2) follow from (5.2), equations (4.3) and (4.4) follow by the definition of the associator. Finally $\Delta$ is an algebra morphism by (5.4) and (5.10). 
5.2. Antipodes of crossed systems. Let $G$ be a group, $(A, \Phi, S, \alpha, \beta)$ be a quasi-Hopf algebra and $\left(\sigma_{*}, \theta_{(\sigma, \tau)}, J_{\sigma}\right)_{\sigma, \tau \in G}$ a $G$-crossed system over $A$. An antipode for $\left(\sigma_{*}, \theta_{(\sigma, \tau)}, J_{\sigma}\right)_{\sigma, \tau \in G}$ is a function $v: G \rightarrow A^{\times}$such that

$$
\begin{gathered}
v_{\sigma \tau}(\sigma \tau)_{*}\left(S\left(\theta_{\sigma, \tau}\right)\right)=v_{\tau}\left(\tau^{-1}\right)_{*}\left(v_{\sigma}\right) \theta_{\tau^{-1}, \sigma^{-1}}, \\
v_{\sigma}^{-1} S(x) v_{\sigma}=\left(\sigma^{-1}\right)_{*}\left(S\left(\sigma_{*}(x)\right)\right), \\
v_{\sigma}\left(\sigma^{-1}\right)_{*}\left(\left(S\left(J_{\sigma}^{1}\right) \alpha J_{\sigma}^{2}\right)\right) \theta_{\sigma^{-1}, \sigma}=\alpha, \\
J_{\sigma}^{1} \sigma_{*}\left(\beta v_{\sigma}\left(\sigma^{-1}\right)_{*}\left(S\left(J_{\sigma}^{2}\right)\right)\right) \theta_{\sigma, \sigma^{-1}}=\beta,
\end{gathered}
$$

for all $\sigma \in G$, where $J_{\sigma}=J_{\sigma}^{1} \otimes J_{\sigma}^{2}$. The next proposition follows by a straightforward verification.

Proposition 5.3. Let $v: G \rightarrow A^{\times}$be an antipode for $\left(\sigma_{*}, \theta_{(\sigma, \tau)}, J_{\sigma}\right)_{\sigma, \tau \in G}$. Then $(S, \alpha \# e, \beta \# e)$ is an antipode for $A \# G$, where

$$
S(x \# \sigma)=v_{\sigma}\left(\sigma^{-1}\right)_{*}(S(x)) \# \sigma^{-1},
$$

for all $\sigma \in G, x \in A$.

5.3. Equivariantization and crossed systems. Let us assume that $G$ is an Abelian group. In this case a $G$-crossed system over $A$ gives rise to a $G$-action on the category $\operatorname{Rep}(A)$. Indeed, for any $\sigma \in G$ we can define the tensor functors $\left(F_{\sigma}, \zeta_{\sigma}\right): \operatorname{Rep}(A) \rightarrow \operatorname{Rep}(A)$ described as follows. For any $V \in \operatorname{Rep}(A), F_{\sigma}(V)=V$ as vector spaces and the action on $F_{\sigma}(V)$ is given by $a \cdot v=\sigma_{*}(a) v$ for all $a \in A, v \in V$. For any $V, W \in \operatorname{Rep}(A)$ the isomorphisms $\left(\zeta_{\sigma}\right)_{V, W}: V \otimes W \rightarrow V \otimes W$ are given by $\left(\zeta_{\sigma}\right)_{V, W}(v \otimes w)=$ $J_{\sigma} \cdot(v \otimes w)$ for all $v \in V, w \in W$. For any $\sigma, \tau \in G$ the natural tensor transformation $\gamma_{\sigma, \tau}: F_{\sigma} \circ F_{\tau} \rightarrow F_{\sigma \tau},\left(\gamma_{\sigma, \tau}\right)_{V}(v)=\theta_{(\sigma, \tau)}^{-1} v$ for all $V \in \operatorname{Rep}(A)$, $v \in V$.

Lemma 5.4. If $\theta_{(\sigma, \tau)}=\theta_{(\tau, \sigma)}$ for all $\sigma, \tau \in G$ then the tensor functors $\left(F_{\sigma}, \zeta_{\sigma}\right)$ described above define a $G$-action on $\operatorname{Rep}(A)$.

Proof. The conmutativity of $G$ and equation $\theta_{(\sigma, \tau)}=\theta_{(\tau, \sigma)}$ for all $\sigma, \tau \in G$ imply that the maps $\gamma_{\sigma, \tau}$ are morphisms of $A$-modules. The proof that the tensor functors $\left(F_{\sigma}, \zeta_{\sigma}\right)$ define a $G$-action is straightforward.

Given a $G$-crossed system $\left(\sigma_{*}, \theta_{(\sigma, \tau)}, J_{\sigma}\right)_{\sigma, \tau \in G}$ over $A$ we consider the category $\operatorname{Rep}(A)^{G}$ of $G$-equivariant $A$-modules.

Proposition 5.5. Let $G$ be an Abelian group, $A$ be a quasi-Hopf algebra and $\left(\sigma_{*}, \theta_{(\sigma, \tau)}, J_{\sigma}\right)_{\sigma, \tau \in G}$ a $G$-crossed system over $A$ such that $\theta_{(\sigma, \tau)}=\theta_{(\tau, \sigma)}$ for all $\sigma, \tau \in G$. Then there is a tensor equivalence between $\operatorname{Rep}(A)^{G}$ and $\operatorname{Rep}(A \# G)$.

Proof. Let $(V, u)$ be a $G$-equivariant object. The linear isomorphisms $u_{\sigma}$ : $F_{\sigma}(V) \rightarrow V$ satisfy

$$
u_{\sigma}\left(\sigma_{*}(a) \cdot v\right)=a \cdot u_{\sigma}(v), \quad u_{\sigma}\left(u_{\tau}(v)\right)=u_{\sigma \tau}\left(\theta_{(\sigma, \tau)} \cdot v\right)
$$


for all $v \in V, a \in A, \sigma, \tau \in G$. Equation (5.15) together with the fact that $\theta_{(\sigma, \tau)}=\theta_{(\tau, \sigma)}$ for all $\sigma, \tau \in G$ imply that there is a well-defined action of the crossed product $A \# G$ on $V$ determined by

$$
(a \# \sigma) \cdot v=a u_{\sigma}^{-1}(v),
$$

for all $a \in A, v \in V, \sigma \in G$. Morphisms of $G$-equivariant representations are exactly morphisms of $A \# G$-modules. Hence we have defined a functor

$$
\mathcal{F}: \operatorname{Rep}(A)^{G} \rightarrow \operatorname{Rep}(A \# G),
$$

which clearly is a tensor functor. Assume that $W \in \operatorname{Rep}(A \# G)$. Then, by restriction, $W$ is a representation of $A$. Moreover $(W, u)$ is a $G$-equivariant object in $\operatorname{Rep}(A)$, letting

$$
u_{\sigma}: W \rightarrow W, \quad u_{\sigma}(w)=\left(\theta_{\left(\sigma, \sigma^{-1}\right)}^{-1} \# \sigma^{-1}\right) \cdot w
$$

for every $\sigma \in G$. We have thus a functor $\mathcal{G}: \operatorname{Rep}(A \# G) \rightarrow \operatorname{Rep}(A)^{G}$. It is clear that $\mathcal{F}$ and $\mathcal{G}$ are inverse equivalences of categories.

Remark 5.6. A version of the above result appears in [Na, Prop. 3.2].

\subsection{Crossed product of quasi-bialgebras.}

Definition 5.7. Let $(A, \Phi, S, \alpha, \beta)$ be a quasi-Hopf algebra, and let $G$ be a group. We shall say that $A$ is a $G$-crossed product if there is a decomposition $A=\bigoplus_{\sigma \in G} A_{\sigma}$, where:

- $\Phi \in A_{e} \otimes A_{e} \otimes A_{e}$,

- $A_{\sigma} A_{\tau} \subseteq A_{\sigma \tau}$ for all $\sigma, \tau \in G$,

- $A_{\sigma}$ has an invertible element for each $\sigma \in G$,

- $\Delta\left(A_{\sigma}\right) \subseteq A_{\sigma} \otimes A_{\sigma}$ for each $\sigma \in G$.

- $S\left(A_{\sigma}\right) \subseteq A_{\sigma^{-1}}$, for each $\sigma \in G$.

- $\alpha, \beta \in A_{e}$

Proposition 5.8. Every $G$-crossed product $A$ is of the form $B \# G$ for some quasi-Hopf algebra $B$. Moreover, there exists an antipode $v: G \rightarrow B^{\times}$such that $B \# G$ is isomorphic to $A$ as quasi-Hopf algebras.

Proof. Let $A$ be a $G$-crossed product. Set $B=A_{e}$. Since every $A_{\sigma}$ has an invertible element, we may choose for each $\sigma \in G$ some invertible element $t_{\sigma} \in A_{\sigma}$, with $t_{e}=1$. Then it is clear that $A_{\sigma}=t_{\sigma} A_{e}=A_{e} t_{\sigma}$, and the set $\left\{t_{\sigma}: \sigma \in G\right\}$ is a basis for $A$ as a left (and right) $A_{e}$-module. Note that $\varepsilon\left(t_{\sigma}\right) \neq 0$, because $\varepsilon$ is an algebra map and $t_{\sigma}$ is invertible. Thus, we may and shall assume that $\varepsilon\left(t_{\sigma}\right)=1$ for each $\sigma \in G$. Let us define the maps

$$
\sigma_{*}(a)=t_{\sigma} a t_{\sigma}^{-1}, \text { for each } \sigma G \text { and } a \in A_{e},
$$

and

$$
\theta: G \times G \rightarrow A \text { by } \theta_{(\sigma, \tau)}=t_{\sigma} t_{\tau} t_{\sigma \tau}^{-1} \text { for } \sigma, \tau \in G .
$$

We have that $\Delta\left(t_{\sigma}\right) \in A_{\sigma} \otimes A_{\sigma}$ can be uniquely expressed as $\Delta\left(t_{\sigma}\right)=$ $J_{\sigma}\left(t_{\sigma} \otimes t_{\sigma}\right)$, with $J_{\sigma} \in A_{e} \otimes A_{e}$. Since $\Delta$ is an algebra morphism, $J_{\sigma}$ is 
invertible, and for the normalization $\varepsilon\left(t_{\sigma}\right)=1,(\varepsilon \otimes \mathrm{id})\left(J_{\sigma}\right)=(\mathrm{id} \otimes \varepsilon)\left(J_{\sigma}\right)=$ 1.

Then, it is straightforward to see that the data $\left(\sigma_{*}, \theta_{(\sigma, \tau)}, J_{\sigma}\right)_{\sigma, \tau \in G}$, defines a $G$-crossed system over the sub-quasi-bialgebra $A_{e} \subseteq A$, and $A_{e} \# G$ is isomorphic to $A$ as quasi-bialgebras.

The antipode $S: A \rightarrow A$ is anti-isomorphism of algebras, and the condition $S\left(A_{\sigma}\right) \subset A_{\sigma^{-1}}$ implies that there is a unique function $v: G \rightarrow A_{e}^{\times}$ such that $S\left(t_{\sigma}\right)=\theta_{\sigma} t_{\sigma^{-1}}$ for all $\sigma \in G$. Hence, it is straightforward to see that $v$ is antipode for the crossed system $\left(\sigma_{*}, \theta_{(\sigma, \tau)}, J_{\sigma}\right)_{\sigma, \tau \in G}$, and $A_{e} \# G$ is isomorphic to $A$ as quasi-Hopf algebras.

5.5. Twisted homomorphisms of comodule algebras. Let $A$ be a quasiHopf algebra. A twisted homomorphism of left $A$-comodule algebras $\left(\mathcal{K}, \lambda, \Phi_{\lambda}\right)$ and $\left(\mathcal{K}^{\prime}, \lambda^{\prime}, \Phi_{\lambda}^{\prime}\right)$ is pair $(\mathfrak{f}, \mathfrak{J})$ consisting of a homomorphism of algebras $\mathfrak{f}: \mathcal{K} \rightarrow \mathcal{K}^{\prime}$ and an invertible element $\mathfrak{J} \in A \otimes \mathcal{K}^{\prime}$ such that

$$
\begin{gathered}
\Phi_{\lambda^{\prime}}(\Delta \otimes \mathrm{id})(\mathfrak{J})=\left(\mathrm{id} \otimes \lambda^{\prime}\right)(\mathfrak{J})(1 \otimes J)(\mathrm{id} \otimes \mathrm{id} \otimes \mathfrak{f})\left(\Phi_{\lambda}\right), \\
(\varepsilon \otimes \mathrm{id})(\mathfrak{J})=1, \\
\lambda^{\prime}(\mathfrak{f}(a)) \mathfrak{J}=\mathfrak{J}(\mathrm{id} \otimes \mathfrak{f})(\lambda(a)), \quad \text { for all } a \in \mathcal{K} .
\end{gathered}
$$

A morphism between two twisted homomorphisms $\left(\mathfrak{f}_{1}, \mathfrak{J}_{1}\right),\left(\mathfrak{f}_{2}, \mathfrak{J}_{2}\right): \mathcal{K} \rightarrow$ $\mathcal{K}^{\prime}$ is an element $c \in \mathcal{K}^{\prime}$ such that $c \mathfrak{f}_{1}(a)=\mathfrak{f}_{2}(a) c$ for any $a \in \mathcal{K}$ and $\lambda^{\prime}(c) \mathfrak{J}_{1}=\mathfrak{J}_{2}(1 \otimes c)$.

To any twisted homomorphism of comodule algebras $(\mathfrak{f}, \mathfrak{J}): \mathcal{K} \rightarrow \mathcal{K}^{\prime}$ there is associated a $\operatorname{Rep}(A)$-module functor

$$
\left(\mathfrak{f}^{*}, \xi^{\mathfrak{J}}\right): \operatorname{Rep}\left(\mathcal{K}^{\prime}\right) \rightarrow \operatorname{Rep}(\mathcal{K}),
$$

where, for all $V \in \operatorname{Rep}\left(\mathcal{K}_{2}\right), \mathfrak{f}^{*}(V)=V$ with action given by $x \cdot v=\mathfrak{f}(x) v$, $x \in \mathcal{K}, v \in V$. The natural transformation $\xi^{\mathfrak{J}}$ is given by

$$
\xi^{\mathfrak{J}}{ }_{X, M}: \mathfrak{f}^{*}(X \otimes M) \rightarrow X \otimes \mathfrak{f}^{*}(M), \quad \xi_{X, M}^{\mathfrak{J}}(x \otimes m)=\mathfrak{J}^{-1} \cdot(x \otimes m),
$$

for any $X \in \operatorname{Rep}(A), M \in \operatorname{Rep}\left(\mathcal{K}_{2}\right), x \in X, m \in M$. Morphisms between twisted homomorphisms $\mathfrak{f}, \mathfrak{f}^{\prime}: \mathcal{K} \rightarrow \mathcal{K}^{\prime}$ of $A$-comodule algebras correspond to module natural transformations between the module functors.

Let $A$ be a quasi-Hopf algebra and $\left(\mathcal{K}, \lambda, \Phi_{\lambda}\right)$ be a left $A$-comodule algebra. For each twisted endomorphism $(f, J): A \rightarrow A$, we define a new left $A$ comodule algebra $\left(\mathcal{K}^{f}, \lambda^{f}, \Phi_{\lambda}^{f}\right)$, where $\mathcal{K}^{f}=\mathcal{K}$ as algebras and

$$
\lambda^{f}(x)=(f \otimes \mathrm{id}) \lambda(x), \quad \Phi_{\lambda}^{f}=(f \otimes f \otimes \mathrm{id})\left(\Phi_{\lambda}\right)\left(J^{-1} \otimes 1\right),
$$

for all $x \in \mathcal{K}$.

Definition 5.9. Let $A$ be a quasi-Hopf algebra and $\left(\mathcal{K}, \lambda, \Phi_{\lambda}\right)$ be a left $A$-comodule algebra. Given a twisted endomorphism $(f, J)$ of $A$, a $(f, J)$ twisted endomorphism of $\mathcal{K}$ is a twisted homomorphism from $\left(\mathcal{K}^{f}, \lambda^{f}, \Phi_{\lambda}^{f}\right)$ 
to $\left(\mathcal{K}, \lambda, \Phi_{\lambda}\right)$. Explicitly a $(f, J)$-twisted endomorphism is a pair $(\mathfrak{f}, \mathfrak{J})$ consisting of an algebra endomorphism $\mathfrak{f}: \mathcal{K} \rightarrow \mathcal{K}$ and an invertible element $\mathfrak{J} \in A \otimes \mathcal{K}$, such that:

$$
\begin{gathered}
(\varepsilon \otimes \mathrm{id})(\mathfrak{J})=1, \\
\Phi_{\lambda}(\Delta \otimes \mathrm{id})(\mathfrak{J})(J \otimes 1)=(\text { id } \otimes \lambda)(\mathfrak{J})(1 \otimes \mathfrak{J})(f \otimes f \otimes \mathfrak{f})\left(\Phi_{\lambda}\right) \\
\lambda(\mathfrak{f}(x)) \mathfrak{J}=\mathfrak{J}(f \otimes \mathfrak{f})(\lambda(x)), \quad \text { for all } x \in \mathcal{K} .
\end{gathered}
$$

Lemma 5.10. Let $\left(\sigma_{*}, \theta_{(\sigma, \tau)}, J_{\sigma}\right)_{\sigma, \tau \in G}$ be a crossed system over a quasi-Hopf algebra $A$, and $\left(\mathcal{K}, \lambda, \Phi_{\lambda}\right)$ a left $A$-comodule algebra. If $\left(\mathfrak{f}_{\sigma}, \mathfrak{J}_{\sigma}\right),\left(\mathfrak{f}_{\tau}, \mathfrak{J}_{\tau}\right)$ : $\mathcal{K} \rightarrow \mathcal{K}$ are $\left(\sigma_{*}, J_{\sigma}\right)$-twisted and $\left(\tau_{*}, J_{\tau}\right)$-twisted endomorphism, then

$$
\left(\mathfrak{f}_{\sigma}, \mathfrak{J}_{\sigma}\right) \bar{\circ}\left(\mathfrak{f}_{\tau}, \mathfrak{J}_{\tau}\right)=\left(\mathfrak{f}_{\sigma} \circ \mathfrak{f}_{\tau}, \mathfrak{J}_{\sigma}\left(\sigma_{*} \otimes \mathfrak{f}_{\sigma}\right)\left(\mathfrak{J}_{\tau}\right)\left(\theta_{\sigma, \tau} \otimes 1\right)\right)
$$

is a $\left((\sigma \tau)_{*}, J_{\sigma \tau}\right)$-twisted endomorphism. Moreover, this composition is associative, i.e., if $\left(\mathfrak{f}_{\sigma}, \mathfrak{J}_{\sigma}\right),\left(\mathfrak{f}_{\tau}, \mathfrak{J}_{\tau}\right),\left(\mathfrak{f}_{\rho}, \mathfrak{J}_{\rho}\right): \mathcal{K} \rightarrow \mathcal{K}$ are $\left(\sigma_{*}, J_{\sigma}\right)$-twisted, $\left(\tau_{*}, J_{\tau}\right)$ twisted, and $\left(\rho_{*}, J_{\rho}\right)$-twisted endomorphism, then

$$
\left[\left(\mathfrak{f}_{\sigma}, \mathfrak{J}_{\sigma}\right) \overline{\bar{\sigma}}\left(\mathfrak{f}_{\tau}, \mathfrak{J}_{\tau}\right)\right] \bar{\sigma}\left(\mathfrak{f}_{\rho}, \mathfrak{J}_{\rho}\right)=\left(\mathfrak{f}_{\sigma}, \mathfrak{J}_{\sigma}\right) \overline{\mathrm{\sigma}}\left[\left(\mathfrak{f}_{\tau}, \mathfrak{J}_{\tau}\right) \overline{\bar{\sigma}}\left(\mathfrak{f}_{\rho}, \mathfrak{J}_{\rho}\right)\right]
$$

Proof. If we use the following notation

$$
\mathfrak{J}_{\sigma} \bar{\varnothing} \mathfrak{J}_{\tau}=\mathfrak{J}_{\sigma}\left(\sigma_{*} \otimes \mathfrak{f}_{\sigma}\right)\left(\mathfrak{J}_{\tau}\right)\left(\theta_{\sigma, \tau} \otimes 1\right),
$$

thus we need to prove:

(1) $(\varepsilon \otimes \mathrm{id})\left(\mathfrak{J}_{\sigma} \bar{\circ} \mathfrak{J}_{\tau}\right)=1$,

(2) $\Phi_{\lambda}(\Delta \otimes \mathrm{id})\left(\mathfrak{J}_{\sigma} \bar{\circ} \mathfrak{J}_{\tau}\right)\left(J_{\sigma \tau} \otimes 1\right)=(\mathrm{id} \otimes \lambda)\left(\mathfrak{J}_{\sigma} \bar{\circ} \mathfrak{J}_{\tau}\right)\left(1 \otimes \mathfrak{J}_{\sigma} \overline{0} \mathfrak{J}_{\tau}\right)\left((\sigma \tau)_{*} \otimes\right.$ $\left.(\sigma \tau)_{*} \otimes \mathfrak{f}_{\sigma} \circ \mathfrak{f}_{\tau}\right)\left(\Phi_{\lambda}\right)$

(3) $\lambda\left(\mathfrak{f}_{\sigma} \circ \mathfrak{f}_{\tau}(x)\right) \mathfrak{J}_{\sigma} \bar{\circ} \mathfrak{J}_{\tau}=\mathfrak{J}_{\sigma} \bar{\sigma} \mathfrak{J}_{\tau}(f \otimes \mathfrak{f})(\lambda(x))$ for all $x \in \mathcal{K}$.

(1) The first equation follows immediately using $\varepsilon \otimes$ id $\left(\mathfrak{J}_{\sigma}\right)=1$, and $\varepsilon$ is an algebra morphism that commutes with $\sigma_{*}$ for all $\sigma \in G$.

(2) For the second equation, first we shall see some equalities:

$(\Delta \otimes \mathrm{id})\left(\sigma_{*} \otimes \mathfrak{f}_{\sigma}\right)\left(\mathfrak{J}_{\tau}\right)\left(J_{\sigma} \otimes 1\right)=\left(J_{\sigma} \otimes 1\right)\left(\sigma_{*} \otimes \sigma_{*} \otimes \mathfrak{f}_{\sigma}\right)(\Delta \otimes \mathrm{id})\left(\mathfrak{J}_{\tau}\right)$

$\left(1 \otimes \mathfrak{J}_{\sigma}\right)\left(\sigma_{*} \otimes \sigma_{*} \otimes \mathfrak{f}_{\sigma}\right)\left[(\mathrm{id} \otimes \lambda)\left(\mathfrak{J}_{\tau}\right)\right]=(\mathrm{id} \otimes \lambda)\left(\sigma_{*} \otimes \mathfrak{f}_{\sigma}\right)\left(\mathfrak{J}_{\tau}\right)\left(1 \otimes \mathfrak{J}_{\sigma}\right)$

The equation (5.23) follows by axiom (5.4) of $J_{\sigma \tau}$, and the equation (5.24) follows by axiom (5.22) of $\mathfrak{J}_{\sigma}$.

$$
\begin{array}{r}
\Phi_{\lambda}(\Delta \otimes \mathrm{id})\left(\mathfrak{J}_{\sigma}\left(\sigma_{*} \otimes \mathfrak{f}_{\sigma}\right)\left(\mathfrak{J}_{\tau}\right)\right)\left(J_{\sigma} \otimes 1\right) \\
=\Phi_{\lambda}(\Delta \otimes \mathrm{id})\left(\mathfrak{J}_{\sigma}\right)(\Delta \otimes \mathrm{id})\left(\sigma_{*} \otimes \mathfrak{f}_{\sigma}\right)\left(\mathfrak{J}_{\tau}\right)\left(J_{\sigma} \otimes 1\right) \\
=(\mathrm{id} \otimes \lambda)\left(\mathfrak{J}_{\sigma}\right)\left(1 \otimes \mathfrak{J}_{\sigma}\right)\left(\sigma_{*} \otimes \sigma_{*} \otimes \mathfrak{f}_{\sigma}\right)\left(\Phi_{\lambda}\right)\left(\sigma_{*} \otimes \sigma_{*} \otimes \mathfrak{f}_{\sigma}\right)(\Delta \otimes \mathrm{id})\left(\mathfrak{J}_{\tau}\right) \\
=(\mathrm{id} \otimes \lambda)\left(\mathfrak{J}_{\sigma}\right)\left(1 \otimes \mathfrak{J}_{\sigma}\right)\left(\sigma_{*} \otimes \sigma_{*} \otimes \mathfrak{f}_{\sigma}\right)\left[\Phi_{\lambda}(\Delta \otimes \mathrm{id})\left(\mathfrak{J}_{\tau}\right)\right]
\end{array}
$$




$$
\begin{aligned}
& (5.26) \quad \Phi_{\lambda}(\Delta \otimes \mathrm{id})\left(\mathfrak{J}_{\sigma}\left(\sigma_{*} \otimes \mathfrak{f}_{\sigma}\right)\left(\mathfrak{J}_{\tau}\right)\right)\left[J_{\sigma}\left(\sigma_{*} \otimes \sigma_{*}\right)\left(J_{\tau}\right) \otimes 1\right] \\
& \underline{5.25)}=(\mathrm{id} \otimes \lambda)\left(\mathfrak{J}_{\sigma}\right)\left(1 \otimes \mathfrak{J}_{\sigma}\right)\left(\sigma_{*} \otimes \sigma_{*} \otimes \mathfrak{f}_{\sigma}\right)\left[\Phi_{\lambda}(\Delta \otimes \mathrm{id})\left(\mathfrak{J}_{\tau}\right)\right]\left(\left(\sigma_{*} \otimes \sigma_{*}\right)\left(J_{\tau}\right)\right) \otimes 1 \\
& =(\mathrm{id} \otimes \lambda)\left(\mathfrak{J}_{\sigma}\right)\left(1 \otimes \mathfrak{J}_{\sigma}\right)\left(\sigma_{*} \otimes \sigma_{*} \otimes \mathfrak{f}_{\sigma}\right)\left[\Phi_{\lambda}(\Delta \otimes \mathrm{id})\left(\mathfrak{J}_{\tau}\right)\left(J_{\tau} \otimes 1\right)\right] \\
& \text { 5.21] }=(\mathrm{id} \otimes \lambda)\left(\mathfrak{J}_{\sigma}\right)\left(1 \otimes \mathfrak{J}_{\sigma}\right)\left(\sigma_{*} \otimes \sigma_{*} \otimes \mathfrak{f}_{\sigma}\right)\left[(\mathrm{id} \otimes \lambda)\left(\mathfrak{J}_{\tau}\right)\left(1 \otimes \mathfrak{J}_{\tau}\right)\left(\tau_{*} \otimes \tau_{*} \otimes \mathfrak{f}_{\tau}\right)\left(\Phi_{\lambda}\right)\right] \\
& =(\mathrm{id} \otimes \lambda)\left(\mathfrak{J}_{\sigma}\right)\left(1 \otimes \mathfrak{J}_{\sigma}\right)\left(\sigma_{*} \otimes \sigma_{*} \otimes \mathfrak{f}_{\sigma}\right)\left[(\mathrm{id} \otimes \lambda)\left(\mathfrak{J}_{\tau}\right)\left(1 \otimes \mathfrak{J}_{\tau}\right)\right]\left(\sigma_{*} \tau_{*} \otimes \sigma_{*} \tau_{*} \otimes \mathfrak{f}_{\sigma} \mathfrak{f}_{\tau}\right)\left(\Phi_{\lambda}\right) \\
& =(\mathrm{id} \otimes \lambda)\left(\mathfrak{J}_{\sigma}\right)\left(1 \otimes \mathfrak{J}_{\sigma}\right)\left(\sigma_{*} \otimes \sigma_{*} \otimes \mathfrak{f}_{\sigma}\right)\left[(\mathrm{id} \otimes \lambda)\left(\mathfrak{J}_{\tau}\right)\right] \\
& \times\left[1 \otimes\left(\sigma_{*} \otimes \mathfrak{f}_{\sigma}\right)\left(\mathfrak{J}_{\tau}\right)\right]\left(\sigma_{*} \tau_{*} \otimes \sigma_{*} \tau_{*} \otimes \mathfrak{f}_{\sigma} \mathfrak{f}_{\tau}\right)\left(\Phi_{\lambda}\right) \\
& \sqrt{5.24}=(\mathrm{id} \otimes \lambda)\left(\mathfrak{J}_{\sigma}\right)(\mathrm{id} \otimes \lambda)\left(\sigma_{*} \otimes \mathfrak{f}_{\sigma}\right)\left(\mathfrak{J}_{\tau}\right)\left(1 \otimes \mathfrak{J}_{\sigma}\right) \\
& \times\left[1 \otimes\left(\sigma_{*} \otimes \mathfrak{f}_{\sigma}\right)\left(\mathfrak{J}_{\tau}\right)\right]\left(\sigma_{*} \tau_{*} \otimes \sigma_{*} \tau_{*} \otimes \mathfrak{f}_{\sigma} \mathfrak{f}_{\tau}\right)\left(\Phi_{\lambda}\right) \\
& =(\mathrm{id} \otimes \lambda)\left[\left(\mathfrak{J}_{\sigma}\right)\left(\sigma_{*} \otimes \mathfrak{f}_{\sigma}\right)\left(\mathfrak{J}_{\tau}\right)\right]\left(1 \otimes \mathfrak{J}_{\sigma}\right) \\
& \times\left[1 \otimes\left(\sigma_{*} \otimes \mathfrak{f}_{\sigma}\right)\left(\mathfrak{J}_{\tau}\right)\right]\left(\sigma_{*} \tau_{*} \otimes \sigma_{*} \tau_{*} \otimes \mathfrak{f}_{\sigma} \mathfrak{f}_{\tau}\right)\left(\Phi_{\lambda}\right) \\
& =(\mathrm{id} \otimes \lambda)\left(\mathfrak{f}_{\sigma} \overline{0} \mathfrak{f}_{\tau}\right)\left(1 \otimes \mathfrak{f}_{\sigma} \overline{0} \mathfrak{f}_{\tau}\right)\left(\theta_{(\sigma, \tau)}^{-1} \otimes \theta_{(\sigma, \tau)}^{-1} \otimes 1\right)\left(\sigma_{*} \tau_{*} \otimes \sigma_{*} \tau_{*} \otimes \mathfrak{f}_{\sigma} \mathfrak{f}_{\tau}\right)\left(\Phi_{\lambda}\right) \\
& \text { [5.7] }=(\mathrm{id} \otimes \lambda)\left(\mathfrak{J}_{\sigma} \bar{\varnothing} \mathfrak{J}_{\tau}\right)\left(1 \otimes \mathfrak{J}_{\sigma} \overline{0} \mathfrak{J}_{\tau}\right)\left((\sigma \tau)_{*} \otimes(\sigma \tau)_{*} \otimes \mathfrak{f}_{\sigma} \mathfrak{f}_{\tau}\right)\left(\Phi_{\lambda}\right)\left(\theta_{(\sigma, \tau)}^{-1} \otimes \theta_{(\sigma, \tau)}^{-1} \otimes 1\right) \\
& \Phi_{\lambda}(\Delta \otimes \mathrm{id})\left(\mathfrak{J}_{\sigma} \overline{\bar{J}} \mathfrak{J}_{\tau}\right)\left(J_{\sigma \tau} \otimes 1\right) \\
& =\Phi_{\lambda}(\Delta \otimes \mathrm{id})\left(\mathfrak{J}_{\sigma}\left(\sigma_{*} \otimes \mathfrak{f}_{\sigma}\right)\left(\mathfrak{J}_{\tau}\right)\left(\theta_{\sigma, \tau} \otimes 1\right)\right)\left(J_{\sigma \tau} \otimes 1\right) \\
& =\Phi_{\lambda}(\Delta \otimes \mathrm{id})\left(\mathfrak{J}_{\sigma}\left(\sigma_{*} \otimes \mathfrak{f}_{\sigma}\right)\left(\mathfrak{J}_{\tau}\right)\right)\left(\Delta\left(\theta_{\sigma, \tau}\right) J_{\sigma \tau} \otimes 1\right) \\
& \text { (5.10) }=\Phi_{\lambda}(\Delta \otimes \mathrm{id})\left(\mathfrak{J}_{\sigma}\left(\sigma_{*} \otimes \mathfrak{f}_{\sigma}\right)\left(\mathfrak{J}_{\tau}\right)\right)\left[\left(J_{\sigma}\left(\sigma_{*} \otimes \sigma_{*}\right)\left(J_{\tau}\right)\left(\theta_{(\sigma, \tau)} \otimes \theta_{(\sigma, \tau)}\right)\right) \otimes 1\right] \\
& =\Phi_{\lambda}(\Delta \otimes \mathrm{id})\left(\mathfrak{J}_{\sigma}\left(\sigma_{*} \otimes \mathfrak{f}_{\sigma}\right)\left(\mathfrak{J}_{\tau}\right)\right)\left[J_{\sigma}\left(\sigma_{*} \otimes \sigma_{*}\right)\left(J_{\tau}\right) \otimes 1\right] \\
& \times\left[\theta_{(\sigma, \tau)} \otimes \theta_{(\sigma, \tau)} \otimes 1\right] \\
& \text { (5.26) }=(\mathrm{id} \otimes \lambda)\left(\mathfrak{J}_{\sigma} \bar{\circ} \mathfrak{J}_{\tau}\right)\left(1 \otimes \mathfrak{f}_{\sigma} \overline{\bar{f}} \tau\right)\left((\sigma \tau)_{*} \otimes(\sigma \tau)_{*} \otimes \mathfrak{f}_{\sigma} \mathfrak{f}_{\tau}\right)\left(\Phi_{\lambda}\right)\left(\theta^{-1} \otimes \theta^{-1} \otimes 1\right) \\
& \times\left[\theta_{(\sigma, \tau)} \otimes \theta_{(\sigma, \tau)} \otimes 1\right] \\
& =(\mathrm{id} \otimes \lambda)\left(\mathfrak{J}_{\sigma} \bar{\sigma} \mathfrak{J}_{\tau}\right)\left(1 \otimes \mathfrak{f}_{\sigma} \bar{\circ} \mathfrak{f}_{\tau}\right)\left((\sigma \tau)_{*} \otimes(\sigma \tau)_{*} \otimes \mathfrak{f}_{\sigma} \mathfrak{f}_{\tau}\right)\left(\Phi_{\lambda}\right)
\end{aligned}
$$

The proof of the second equation is over.

(3) Now we shall prove the third equation:

$$
\begin{aligned}
& \lambda\left(\mathfrak{f}_{\sigma} \circ \mathfrak{f}_{\tau}(x)\right) \mathfrak{J}_{\sigma} \bar{\sigma} \mathfrak{J}_{\tau} \\
&= \lambda\left(\mathfrak{f}_{\sigma} \circ \mathfrak{f}_{\tau}(x)\right) \mathfrak{J}_{\sigma}\left(\sigma_{*} \otimes \mathfrak{f}_{\sigma}\right)\left(\mathfrak{J}_{\tau}\right)\left(\theta_{\sigma, \tau} \otimes 1\right) \\
&= \mathfrak{J}_{\sigma}\left(\sigma_{*} \otimes \mathfrak{f}_{\sigma}\right) \lambda\left(\mathfrak{f}_{\tau}(x)\right)\left(\sigma_{*} \otimes \mathfrak{f}_{\sigma}\right)\left(\mathfrak{J}_{\tau}\right)\left(\theta_{\sigma, \tau} \otimes 1\right) \\
&= \mathfrak{J}_{\sigma}\left(\sigma_{*} \otimes \mathfrak{f}_{\sigma}\right)\left[\lambda\left(\mathfrak{f}_{\tau}(x)\right) \mathfrak{J}_{\tau}\right]\left(\theta_{\sigma, \tau} \otimes 1\right) \\
&=\mathfrak{J}_{\sigma}\left(\sigma_{*} \otimes \mathfrak{f}_{\sigma}\right)\left[\mathfrak{J}_{\tau}\left(\tau_{*} \otimes \mathfrak{f}_{\tau}\right) \lambda(x)\right]\left(\theta_{\sigma, \tau} \otimes 1\right) \\
&=\mathfrak{J}_{\sigma}\left(\sigma_{*} \otimes \mathfrak{f}_{\sigma}\right)\left(\mathfrak{J}_{\tau}\right)\left(\sigma_{*} \tau_{*} \otimes \mathfrak{f}_{\sigma} \mathfrak{f}_{\tau}\right)(\lambda(x))\left(\theta_{\sigma, \tau} \otimes 1\right) \\
&=\mathfrak{J}_{\sigma}\left(\sigma_{*} \otimes \mathfrak{f}_{\sigma}\right)\left(\mathfrak{J}_{\tau}\right)\left(\theta_{\sigma, \tau} \otimes 1\right) \\
&=\left((\sigma \tau)_{*} \otimes \mathfrak{f}_{\sigma} \mathfrak{f}_{\tau}\right)(\lambda(x)) \\
&\left.=\left(\mathfrak{J}_{\sigma} \overline{0} \mathfrak{J}_{\tau}\right)\left((\sigma \tau)_{*} \otimes \mathfrak{f}_{\sigma} \mathfrak{f}_{\tau}\right) \lambda(x)\right]
\end{aligned}
$$


Finally, we shall prove the associativity of $\bar{\sigma}$,

$$
\begin{aligned}
& {\left[\mathfrak{J}_{\sigma} \bar{\circ} \mathfrak{J}_{\tau}\right] \overline{0} \mathfrak{J}_{\rho}=\left[\mathfrak{J}_{\sigma}\left(\sigma_{*} \otimes \mathfrak{f}_{\sigma}\right)\left(\mathfrak{J}_{\tau}\right)\left(\theta_{\sigma, \tau} \otimes 1\right)\right] \bar{\circ} \mathfrak{J}_{\rho}} \\
& =\mathfrak{J}_{\sigma}\left(\sigma_{*} \otimes \mathfrak{f}_{\sigma}\right)\left(\mathfrak{J}_{\tau}\right)\left(\theta_{\sigma, \tau} \otimes 1\right)\left((\sigma \tau)_{*} \otimes\left(\mathfrak{f}_{\sigma} \circ \mathfrak{f}_{\tau}\right)\right)\left(\mathfrak{J}_{\rho}\right)\left(\theta_{\sigma \tau, \rho} \otimes 1\right) \\
& \text { [5.7) }=\mathfrak{J}_{\sigma}\left(\sigma_{*} \otimes \mathfrak{f}_{\sigma}\right)\left(\mathfrak{J}_{\tau}\right)\left(\sigma_{*} \tau_{*} \otimes\left(\mathfrak{f}_{\sigma} \circ \mathfrak{f}_{\tau}\right)\right)\left(\mathfrak{J}_{\rho}\right)\left(\theta_{\sigma, \tau} \theta_{\sigma \tau, \rho} \otimes 1\right) \\
& {[5.8)=\mathfrak{J}_{\sigma}\left(\sigma_{*} \otimes \mathfrak{f}_{\sigma}\right)\left(\mathfrak{J}_{\tau}\right)\left(\sigma_{*} \tau_{*} \otimes\left(\mathfrak{f}_{\sigma} \circ \mathfrak{f}_{\tau}\right)\right)\left(\mathfrak{J}_{\rho}\right)\left(\sigma_{*}\left(\theta_{\tau, \rho}\right) \theta_{\sigma, \tau \rho} \otimes 1\right)} \\
& =\mathfrak{J}_{\sigma}\left(\sigma_{*} \otimes \mathfrak{f}_{\sigma}\right)\left[\mathfrak{J}_{\tau}\left(\tau_{*} \otimes \mathfrak{f}_{\tau}\right)\left(\mathfrak{J}_{\rho}\right)\left(\theta_{\tau, \rho} \otimes 1\right)\right]\left(\theta_{\sigma, \tau \rho} \otimes 1\right) \\
& =\mathfrak{J}_{\sigma}\left(\sigma_{*} \otimes \mathfrak{f}_{\sigma}\right)\left[\mathfrak{J}_{\tau} \bar{\circ} \mathfrak{J}_{\rho}\right]\left(\theta_{\sigma, \tau \rho} \otimes 1\right)=\mathfrak{J}_{\sigma} \bar{\circ}\left[\mathfrak{J}_{\tau} \bar{\circ} \mathfrak{J}_{\rho}\right] .
\end{aligned}
$$

5.6. Crossed system of comodule algebras. Let $A$ be a quasi-Hopf algebra $\left(\mathcal{K}, \lambda, \Phi_{\lambda}\right)$ be a left $A$-comodule algebra and $\left(\sigma_{*}, \theta_{(\sigma, \tau)}, J_{\sigma}\right)_{\sigma, \tau \in G}$ a $G$ crossed system over $A$.

We define the monoidal category $\underline{\mathrm{Aut}}_{G}^{\mathrm{Tw}}(\mathcal{K})$ of twisted automorphisms as follows. Objects in $\underline{\mathrm{Aut}}_{G}^{\mathrm{Tw}}(\mathcal{K})$ are $\left(\sigma_{*}, J_{\sigma}\right)$-twisted automorphisms of $\mathcal{K}$ for $\sigma \in G$, the set of arrows are the isomorphisms of twisted homomorphisms of $A$-comodule algebras, the tensor product of object is defined by the composition explained in Lemma 5.10. The unity object is the $\mathrm{id} \mathcal{K}$, and tensor product of arrows is as in $\underline{\mathrm{Aut}}^{\mathrm{Tw}}(A)$.

Let $F \subset G$ be a subgroup. An $F$-crossed system for a left $A$-comodule algebra $\mathcal{K}$, compatible with the $G$-crossed system $\left(\sigma_{*}, \theta_{(\sigma, \tau)}, J_{\sigma}\right)_{\sigma, \tau \in G}$ is a monoidal functor $\overline{()}: \underline{F} \rightarrow \underline{\operatorname{Aut}}_{G}^{\mathrm{Tw}}(\mathcal{K})$, that is, an $F$-crossed system consists of the following data:

- A $\left(\sigma_{*}, J_{\sigma}\right)$-twisted automorphism $\left(\bar{\sigma}, \overline{J_{\sigma}}\right)$ for each $\sigma \in F$,

- an element $\overline{\theta_{(\sigma, \tau)}} \in \mathcal{K}^{\times}$for each $\sigma, \tau \in F$,

such that

$$
\begin{aligned}
\left(\overline{1}, \overline{J_{1}}\right) & =(\mathrm{id}, 1 \otimes 1), \\
\bar{\theta}_{(\sigma, \tau)} \overline{(\sigma \tau)}(k) & =\bar{\sigma}(\bar{\tau}(k)) \bar{\theta}_{(\sigma, \tau)}, \\
\bar{\theta}_{(\sigma, \tau)} \bar{\theta}_{(\sigma \tau, \rho)} & =\bar{\sigma}\left(\bar{\theta}_{(\tau, \rho)}\right) \bar{\theta}_{\sigma, \tau \rho}, \\
\bar{\theta}_{(1, \sigma)} & =\bar{\theta}_{(\sigma, 1)}=1, \\
\lambda\left(\bar{\theta}_{(\sigma, \tau)}\right) \bar{J}_{\sigma \tau} & =\bar{J}_{\sigma}\left(\left(\sigma_{*} \otimes \bar{\sigma}\right)\left(\bar{J}_{\tau}\right)\right) \theta_{(\sigma, \tau)} \otimes \bar{\theta}_{(\sigma, \tau)},
\end{aligned}
$$

for all $k \in \mathcal{K}, \sigma, \tau, \rho \in F$. Let $\mathcal{K} \# F$ be the vector space $\mathcal{K} \otimes_{\mathbb{k}} \mathbb{k} F$ with product and coaction given by

$$
(x \# \sigma)(y \# \tau)=x \bar{\sigma}(y) \bar{\theta}_{(\sigma, \tau)} \# \sigma \tau, \quad \delta(x \# \sigma)=x_{(-1)} \bar{J}_{\sigma}^{1} \# \sigma \otimes x_{(0)} \bar{J}_{\sigma}^{2} \# \sigma,
$$

for all $x, y \in \mathcal{K}, \sigma, \tau \in F$.

Proposition 5.11. The foregoing operations make the space $\mathcal{K} \# F$ into a left $A \# G$-comodule algebra with associator $\Phi_{\delta}=\Phi_{\lambda}^{1} \# 1 \otimes \Phi_{\lambda}^{2} \# 1 \otimes \Phi_{\lambda}^{3} \# 1$. 
Definition 5.12. Let $G$ be a group, and $F \subseteq G$ be a subgroup. Let $A$ be a $G$-crossed product quasi-bialgebra, and let $\left(\mathcal{K}, \lambda, \Phi_{\lambda}\right)$ be a left $A$-comodule algebra. We shall say that $\mathcal{K}$ is an $F$-crossed product, if there is a decomposition $\mathcal{K}=\bigoplus_{\sigma \in F} \mathcal{K}_{\sigma}$, such that

- $\Phi_{\lambda} \in A_{e} \otimes A_{e} \otimes \mathcal{K}_{e}$,

- $\mathcal{K}_{\sigma} \mathcal{K}_{\tau} \subseteq \mathcal{K}_{\sigma \tau}$ for all $\sigma, \tau \in F$,

- $\mathcal{K}_{\sigma}$ has an invertible element for each $\sigma \in F$,

- $\lambda\left(\mathcal{K}_{\sigma}\right) \subseteq A_{\sigma} \otimes \mathcal{K}_{\sigma}$ for each $\sigma \in F$.

Let $A$ be a quasi-Hopf algebra and $\left(\sigma_{*}, \theta_{(\sigma, \tau)}, J_{\sigma}\right)_{\sigma, \tau \in G}$ be a crossed system for the group $G$. We have similar results as for quasi-Hopf algebras. The proof is analogous to the proof of Proposition 5.8.

Proposition 5.13. Let $(\mathcal{L}, \delta)$ be a $F$-crossed $A \# G$-comodule algebra, for a subgroup $F \subseteq G$. Then there is an $A$-comodule algebra $\mathcal{K}$, and an $F$-crossed system over $\mathcal{K}$ compatible with the crossed system $\left(\sigma_{*}, \theta_{(\sigma, \tau)}, F_{\sigma}\right)_{\sigma, \tau \in G}$, such that $\mathcal{K} \# F$ and $\mathcal{L}$ are isomorphic $A \# G$-comodule algebras.

Proof. Let $\mathcal{L}$ be a $F$-crossed $A \# G$-comodule algebra, for a subgroup $F \subseteq G$. Set $\mathcal{K}=\mathcal{L}_{e}$. Since every $\mathcal{L}_{\sigma}$ has an invertible element, we may choose for each $\sigma \in F$ some invertible element $u_{\sigma} \in \mathcal{L}_{\sigma}$, with $u_{e}=1$. Then it is clear that $\mathcal{L}_{\sigma}=u_{\sigma} \mathcal{L}_{e}=\mathcal{L}_{e} u_{\sigma}$, and the set $\left\{u_{\sigma}: \sigma \in F\right\}$ is a basis for $\mathcal{L}$ as a left (and right) $\mathcal{L}_{e}$-module. Let us define the maps

$$
\bar{\sigma}(a)=u_{\sigma} a u_{\sigma}^{-1}, \text { for each } \sigma F \text { and } a \in \mathcal{L}_{e},
$$

and

$$
\bar{\theta}: G \times G \rightarrow \mathcal{L}_{e} \quad \text { by } \bar{\theta}_{(\sigma, \tau)}=u_{\sigma} u_{\tau} u_{\sigma \tau}^{-1} \text { for } \sigma, \tau \in F .
$$

Note that $\left\{(1 \# \sigma) \otimes u_{\tau}\right\}_{\sigma \in G, \tau \in F}$ is a basis for $A \# F \otimes \mathcal{L}$ as a left (and right) $A \otimes \mathcal{L}_{e}$-module. We have that $\delta\left(u_{\sigma}\right) \in A \# \sigma \otimes \mathcal{L}_{\sigma}$ can be uniquely expressed as $\delta\left(u_{\sigma}\right)=\bar{J}_{\sigma}\left((1 \# \sigma) \otimes u_{\sigma}\right)$, with $\bar{J}_{\sigma} \in A \otimes \mathcal{L}_{e}$, for all $\sigma \in F$.

Then, it is straightforward to see that the data $\left(\bar{\sigma}_{\bar{\theta}} \bar{\theta}_{(\sigma, \tau)}, \bar{J}_{\sigma}\right)_{\sigma, \tau \in F}$, define an $F$-crossed system over the $A$-comodule algebra $\mathcal{L}_{e}$, and $\mathcal{L}_{e} \# F$ is isomorphic to $\mathcal{L}$ as $A \# G$ comodule algebras.

Let $G$ be an Abelian group, $F \subseteq G$ a subgroup, $\left(\sigma_{*}, \theta_{(\sigma, \tau)}, J_{\sigma}\right)_{\sigma, \tau \in G}$ be a crossed system over a quasi-Hopf algebra $A$, and $\left(\bar{\sigma}, \bar{\theta}_{(\sigma, \tau)}, \bar{J}_{\sigma}\right)_{\sigma, \tau \in F}$ be an $F$-crossed system for a $A$-comodule algebra $\mathcal{K}$. We shall further assume that

$$
\theta_{(\sigma, \tau)}=\theta_{(\tau, \sigma)}, \quad \bar{\theta}_{(\rho, \nu)}=\bar{\theta}_{(\nu, \rho)}
$$

for all $\sigma, \tau \in G, \rho, \nu \in F$. We can consider the action of $G$ on the category $\operatorname{Rep}(A)$ described in Lemma 5.4 .

Proposition 5.14. Under the above assumptions the following assertions hold.

1. The $\operatorname{Rep}(A)$-module category $\mathcal{K} \mathcal{M}$ is $F$-equivariant.

2. There is an equivalence between $(\mathcal{K} \mathcal{M})^{F}$ and ${ }_{\mathcal{K} \# F} \mathcal{M}$ as $\operatorname{Rep}(A)^{G}$ module categories. 
Proof. 1. For any $\rho \in F$ define $\left(U_{\rho}, c^{\rho}\right): \mathcal{K} \mathcal{M} \rightarrow(\mathcal{K} \mathcal{M})^{\rho}$ the $\operatorname{Rep}(A)$-module functor given as follows. For any $M \in \mathcal{K} \mathcal{M}, U_{\rho}(M)=M$ as vector spaces and the action of $\mathcal{K}$ is given by: $x \cdot v=\bar{\rho}(x) \cdot v$, for all $x \in \mathcal{K}, v \in M$. For any $X \in \operatorname{Rep}(A), M \in \mathcal{K} \mathcal{M}$ the maps $c_{X, M}^{\rho}: U_{\rho}\left(X \otimes_{\mathbb{k}} M\right) \rightarrow F_{\rho}(X) \otimes_{\mathbb{k}} U_{\rho}(M)$ are defined by $c_{X, M}^{\rho}(x \otimes v)=\bar{J}_{\rho}^{-1} \cdot(x \otimes v)$, for any $x \in X, v \in M$. Equation (2.1) for the pair $\left(U_{\rho}, c^{\rho}\right)$ follows from (5.21).

For any $\sigma, \tau \in F$ define $\mu_{\sigma, \tau}: U_{\sigma} \circ U_{\tau}, \rightarrow U_{\sigma \tau}$ as follows. For any $M \in \mathcal{K} \mathcal{M}$, $m \in M$

$$
\mu_{\sigma, \tau}(m)=\bar{\theta}_{(\sigma, \tau)}^{-1} \cdot m
$$

It follows from equation (5.7) that $\mu_{\sigma, \tau}$ is a morphism of $\mathcal{K}$-modules. Equation (3.2) follows from (5.7) and (3.3) follows from (5.10).

2. Let $\mathcal{T}:(\mathcal{K} \mathcal{M})^{F} \rightarrow{ }_{\mathcal{K} \# F} \mathcal{M}$ be the module functor defined as follows. If $(M, v)$ is an $F$-equivariant object then for any $\sigma \in F$ we have isomorphisms $v_{\sigma}: U_{\sigma}(M) \rightarrow M$ satisfying

$$
v_{\sigma \tau}\left(\theta_{(\sigma, \tau)}^{-1} \cdot m\right)=v_{\sigma}\left(v_{\tau}(m), \quad v_{\sigma}(\bar{\sigma}(x) \cdot m)=x \cdot v_{\sigma}(m)\right.
$$

for all $\sigma, \tau \in F, x \in \mathcal{K}, m \in M$. In this case there is a well-defined action of $\mathcal{K} \# F$ on $M$ determined by

$$
(x \# \sigma) \cdot m=x \cdot v_{\sigma}^{-1}(m),
$$

for all $\sigma \in F, x \in \mathcal{K}, m \in M$. We define $\mathcal{T}(M)=M$ with the above described action. If $(X, u) \in \operatorname{Rep}(A)^{G},(M, v) \in(\mathcal{K} \mathcal{M})^{F}$ the action of $\mathcal{K} \# F$ on $X \otimes M$ using the coaction given in (5.32) coincides with the action (5.34) using the isomorphism $\widetilde{v}$ described in Lemma 3.3. The proof that $\mathcal{T}$ is an equivalence is analogous to the proof of Proposition 5.5.

The category of $F$-equivariant objects in a module category is always of the form ${ }_{\mathcal{K} \# F} \mathcal{M}$ for some left $A$-comodule algebra $\mathcal{K}$ and some group $F$.

Proposition 5.15. Let $A$ be a finite dimensional quasi-Hopf algebra and $G$ be a finite Abelian group and $F \subset G$ a subgroup. Let $\left(\sigma_{*}, \theta_{(\sigma, \tau)}, J_{\sigma}\right)_{\sigma, \tau \in G}$ be a $G$-crossed system over $A$, and $\mathcal{M}$ be an exact $F$-equivariant $\operatorname{Rep}(A)$ module category. Then there is a left $A$-comodule algebra $\left(\mathcal{K}, \lambda, \Phi^{\lambda}\right)$ such that $\mathcal{K} M \cong \mathcal{M}$ as $\operatorname{Rep}(A)$-module categories and there is an $F$-crossed system compatible with $\left(\sigma_{*}, \theta_{(\sigma, \tau)}, J_{\sigma}\right)_{\sigma, \tau \in G}$ such that $\mathcal{K}_{F} \mathcal{M} \simeq \mathcal{M}^{F}$ as $\operatorname{Rep}(A)^{G}$ module categories.

Proof. Let $B$ be a finite-dimensional quasi-Hopf algebra such that there is a quasi-Hopf algebra projection $\pi: B \rightarrow A$ and an equivalence $\operatorname{Rep}(B) \simeq$ $\operatorname{Rep}(A) \rtimes F$ of tensor categories, see section 3.4 . Since $\mathcal{M}$ is $F$-equivariant follows from Proposition 3.4 that $\mathcal{M}$ is an exact $\operatorname{Rep}(B)$ module category.

Hence there exists a left $B$-comodule algebra $\left(\mathcal{K}, \lambda, \Phi^{\lambda}\right)$ such that $\mathcal{M} \simeq$ $\mathcal{K} \mathcal{M}$ as $\operatorname{Rep}(B)$-modules. Let us recall that the equivariant structure is given by

$$
\left(U_{\sigma}, c^{\sigma}\right): \mathcal{M} \rightarrow \mathcal{M}^{\sigma}, \quad U_{\sigma}(M)=[\mathbf{1}, \sigma] \bar{\otimes} M
$$


for all $\sigma \in F, M \in \mathcal{M}$ together with a family of natural isomorphisms $\mu_{\sigma, \tau}: U_{\sigma} \circ U_{\tau} \rightarrow U_{\sigma \tau}$ for any $\sigma, \tau \in F$. Under the equivalence $\operatorname{Rep}(B) \simeq$ $\operatorname{Rep}(A) \rtimes F$ the object $[\mathbf{1}, \sigma]$ correspond to a 1-dimensional representation of $B$. For any $\sigma \in F$ let us denote by $\chi_{\sigma}: B \rightarrow \mathbb{k}$ the corresponding character and the algebra map $\bar{\sigma}: \mathcal{K} \rightarrow \mathcal{K}, \bar{\sigma}(k)=\chi_{\sigma}\left(k_{(-1)}\right) k_{(0)}$, for all $k \in \mathcal{K}$.

Define $\lambda^{\pi}=(\pi \otimes$ id $) \lambda$, then $\left(\mathcal{K}, \lambda^{\pi},(\pi \otimes \pi \otimes\right.$ id $\left.)\left(\Phi^{\lambda}\right)\right)$ is a left $A$-comodule algebra that we will denote by $\mathcal{K}^{\pi}$. The equivalence $\mathcal{M} \simeq \mathcal{K} \mathcal{M}$ of $\operatorname{Rep}(B)$ module categories induces an equivalence $\mathcal{M} \simeq \mathcal{K}^{\pi} \mathcal{M}$ of $\operatorname{Rep}(A)$-modules. Under this equivalence the functors $U_{\sigma}: \mathcal{K}^{\pi} \mathcal{M} \rightarrow\left(\mathcal{K}^{\pi} \mathcal{M}\right)^{\sigma}$ are given as follows. For any $M \in \mathcal{K}^{\pi} \mathcal{M}, U_{\sigma}(M)=M$ and the action of $\mathcal{K}$ on $M$ is given by

$$
k \cdot m=\bar{\sigma}(k) \cdot m, \quad \text { for all } k \in \mathcal{K}, m \in M .
$$

For any $\sigma, \tau \in F$ denote

$$
\bar{J}_{\sigma}=c_{A, \mathcal{K}}^{\sigma}(1 \otimes 1)^{-1}, \quad \bar{\theta}_{\sigma, \tau}=\left(\mu_{\tau, \sigma}\right)_{\mathcal{K}}(1)^{-1} .
$$

Turns out that the collection $\left(\bar{\sigma}_{\bar{\theta}} \bar{\theta}_{(\sigma, \tau)}, \bar{J}_{\sigma}\right)_{\sigma, \tau \in F}$ is an $F$-crossed system compatible with $\left(\sigma_{*}, \theta_{(\sigma, \tau)}, J_{\sigma}\right)_{\sigma, \tau \in G}$ for the $A$-comodule algebra $\mathcal{K}^{\pi}$. Indeed for any $\sigma \in F$ the pair $\left(\bar{\sigma}, \overline{J_{\sigma}}\right)$ is a $\left(\sigma_{*}, J_{\sigma}\right)$-twisted automorphism since equation (5.21) follows from the fact that $c^{\sigma}$ satisfies (2.1) and equation (5.22) follows since $c^{\sigma}$ is a $\mathcal{K}$-module morphism. Equation (5.28) follows since $\mu_{\sigma, \tau}$ is a morphism of $\mathcal{K}$-modules, equation (5.29) follows from (3.2) and equation (5.31) follows from (3.3). The equivalence $\mathcal{K}^{\pi} \# F \mathcal{M} \simeq \mathcal{M}^{F}$ as $\operatorname{Rep}(A)^{G_{-}}$ module categories follows from Proposition 5.14

\section{Module Categories over the Quasi-Hopf algebras $A(H, s)$}

6.1. Basic Quasi-Hopf algebras $A(H, s)$. We recall the definition of a the family of basic quasi-Hopf algebras $A(H, s)$ introduced by I. Angiono [A] and used to give a classification of pointed tensor categories with cyclic group of invertible objects of order $m$ such that $210 \nmid m$.

Let $m \in \mathbb{N}$ and $H=\oplus_{n \geq 0} H(n)$ be a finite-dimensional radically graded pointed Hopf algebra generated by a group like element $\chi$ of order $m^{2}$ and skew primitive elements $x_{1}, \ldots, x_{\theta}$ satisfying

$$
\chi x_{i} \chi^{-1}=q^{d_{i}} x_{i}, \quad \Delta\left(x_{i}\right)=x_{i} \otimes 1+\chi^{-b_{i}} \otimes x_{i},
$$

for any $i=1, \ldots, \theta$, where $q$ is a primitive root of 1 of order $m^{2}, H=$ $\mathfrak{B}(V) \# \mathbb{k} C_{m^{2}}$, where $\mathfrak{B}(V)$ is the associated Nichols algebra of the YetterDrinfeld module $V \in \underset{\mathbb{k}^{2} C_{m^{2}}}{\mathbb{R} C_{m^{2}}} \mathcal{Y} \mathcal{D}$.

We shall further assume that $\mathfrak{B}(V)$ has a basis $\left\{x_{1}^{s_{1}} \ldots x_{\theta}^{s_{\theta}}: 0 \leq s_{i} \leq N_{i}\right\}$.

Remark 6.1. The above condition does not hold for any Nichols algebra. If $V$ has diagonal braiding with Cartan matrix of type $A_{3}$ then $\mathfrak{B}(V)$ is not generated by elements of degree 1 . This conditions is satisfied for example for any quantum linear space. 
Set $\sigma:=\chi^{m}$ and denote by $\left\{1_{i}: i \in C_{m^{2}}\right\},\left\{\mathbf{1}_{j}: j \in C_{m}\right\}$ the families of primitive idempotents in $\mathbb{k} C_{m^{2}}$ and $\mathbb{k} C_{m}$ respectively. That is

$$
1_{i}=\frac{1}{m^{2}} \sum_{k=0}^{m^{2}-1} q^{-k i} \chi^{k}, \quad \mathbf{1}_{j}=\frac{1}{m} \sum_{l=0}^{m-1} q^{-m l j} \sigma^{l} .
$$

For any $0 \leq s \leq m-1$ set $J_{s}=\sum_{i, j=0}^{m^{2}-1} c(i, j)^{s} 1_{i} \otimes 1_{j}$, where $c(i, j):=$ $q^{j\left(i-i^{\prime}\right)}$. Here $j^{\prime}$ denotes the remainder in the division by $m$. The associator $\Phi_{s}=d J_{s}$ is written explicitly as

$$
\Phi_{s}:=\sum_{i, j, k=0}^{m-1} \omega_{s}(i, j, k) \mathbf{1}_{i} \otimes \mathbf{1}_{j} \otimes \mathbf{1}_{k},
$$

where $\omega_{s}:\left(C_{m}\right)^{3} \rightarrow \mathbb{k}^{\times}$is the 3 -cocycle defined by $\omega_{s}(i, j, k)=q^{s k\left(j+i-(j+i)^{\prime}\right)}$. Consider the quasi-Hopf algebra $\left(H_{J_{s}}, \Phi_{s}\right)$ obtained by twisting $H$. Denote $\Upsilon(H)=\left\{1 \leq s \leq m-1: b_{i} \equiv s d_{i} \bmod (m), 1 \leq i \leq \theta\right\}$. For any $s \in \Upsilon(H)$ the quasi-Hopf algebra $A(H, s)$ is defined as the subalgebra of $H$ generated by $\sigma$ and $x_{1}, \ldots, x_{\theta}$. The algebra $A(H, s)$ is a quasi-Hopf subalgebra of $H_{J_{s}}$ with associator $\Phi_{s}$ such that $A(H, s) / \operatorname{Rad} A(H, s) \cong \mathbb{k}\left[C_{m}\right]$. See [A, Prop. 3.1.1].

For any $1 \leq i \leq \theta$ we have that

$$
\begin{aligned}
\Delta_{J_{s}}\left(x_{i}\right) & =\sum_{y=0}^{m-1} q^{b_{i} y} \mathbf{1}_{y} \otimes x_{i}+\sum_{z=0}^{m-1}\left(\sum_{y=0}^{m-d_{i}^{\prime}-1} q^{\left(d_{i}^{\prime}-d_{i}\right) s z} x_{i} \mathbf{1}_{y} \otimes \mathbf{1}_{z}\right. \\
& \left.+\sum_{j=m-d_{i}^{\prime}}^{m-1} q^{\left(d_{i}^{\prime}+m-d_{i}\right) s z} x_{i} \mathbf{1}_{y} \otimes \mathbf{1}_{z}\right) .
\end{aligned}
$$

Remark 6.2. Our definition of $A(H, s)$ is slightly different that the one given in $[\mathrm{A}, \S 3]$. This is not a problem since our quasi-Hopf algebras are isomorphic to the ones defined in loc. cit. except that the $s$ may change. The difference comes from the fact that we are using $\left(\chi^{-b_{i}}, 1\right)$ skew-primitive elements instead of $\left(1, \chi^{b_{i}}\right)$ skew-primitive elements.

6.2. $C_{m}$-crossed system over $A(H, s)$. The cyclic group with $m$ elements will be denoted by $C_{m}=\left\{1, h, h^{2}, \ldots, h^{m-1}\right\}$. For any $0 \leq i<m$ set $\left(\left(h^{i}\right)_{*}, J_{h^{i}}\right)$ the twisted endomorphism of $A(H, s)$ given by

$$
J_{h^{i}}=1 \otimes 1, \quad\left(h^{i}\right)_{*}(a)=\chi^{i^{\prime}} a \chi^{-i^{\prime}} \quad \text { for all } a \in A(H, s) .
$$

For any $0 \leq i, j<m$ define $\theta_{(i, j)}=\theta_{\left(h^{i}, h^{j}\right)}=\sigma^{\frac{(i+j)-(i+j)^{\prime}}{m}}$.

Remark 6.3. If $i+j \leq m$ then $\theta_{(i, j)}=1$ and if $i+j>m$ then $\theta_{(i, j)}=\sigma$. In principle the algebra maps $\left(h^{i}\right)_{*}$ are defined in $H$ but when restricted to $A(H, s)$ they are well-defined. 
These data is a $C_{m}$-crossed system over $A(H, s)$ such that the equivariantization $\operatorname{Rep}(A)^{C_{m}}$ is tensor equivalent to $\operatorname{Rep}(H)$. This is contained in the next result which gives an alternative proof for [A, Thm. 4.2.1].

Proposition 6.4. 1. $\left(\left(h^{i}\right)_{*}, \theta_{(i, j)}, J_{h^{i}}\right)_{h^{i}, h^{j} \in C_{m}}$ is a $C_{m^{-}}$-crossed system over $A(H, s)$.

2. There is an isomorphism of quasi-Hopf algebras $A(H, s) \# C_{m} \simeq H_{J_{s}}$.

3. There is a tensor equivalence $\operatorname{Rep}(A)^{C_{m}} \simeq \operatorname{Rep}(H)$.

Proof. 1. it follows by a straightforward computation.

2. Define $\varphi: A(H, s) \# C_{m} \rightarrow H_{J_{s}}$ the linear map given by

$$
\varphi\left(a \# h^{i}\right)=a \chi^{i^{\prime}},
$$

for all $0 \leq i<m, a \in A$. Let $0 \leq i, j<m, a, b \in A$ then

$$
\varphi\left(\left(a \# h^{i}\right)\left(b \# h^{j}\right)\right)=\varphi\left(a\left(h^{i}\right)_{*}(b) \theta_{(i, j)} \# h^{i+j}\right)=a \chi^{i^{\prime}} b \chi^{-i^{\prime}} \theta_{(i, j)} \chi^{(i+j)^{\prime}} .
$$

On the other hand

$$
\varphi\left(a \# h^{i}\right) \varphi\left(b \# h^{j}\right)=a \chi^{i^{\prime}} b \chi^{j^{\prime}} .
$$

It is enough to prove that $\chi^{i^{\prime}} b \chi^{j^{\prime}}=\chi^{i^{\prime}} b \chi^{-i^{\prime}} \theta_{(i, j)} \chi^{(i+j)^{\prime}}$ for $b=x_{l}, 1 \leq l \leq \theta$. If $i+j \leq m$ then

$$
\chi^{i^{\prime}} x_{l} \chi^{-i^{\prime}} \theta_{(i, j)} \chi^{(i+j)^{\prime}}=q^{d_{l} i} x_{l} \chi^{i+j}=\chi^{i} x_{l} \chi^{j} .
$$

If $i+j=m+k, k>0$ then

$$
\chi^{i^{\prime}} x_{l} \chi^{-i^{\prime}} \theta_{(i, j)} \chi^{(i+j)^{\prime}}=q^{d_{l} i} x_{l} \sigma \chi^{k}=q^{d_{l} i} x_{l} \chi^{i+j}=\chi^{i} x_{l} \chi^{j} .
$$

It follows immediately that $\varphi$ is a coalgebra map and it is injective and by a dimension argument is bijective.

3. It follows from Proposition 5.5.

Remark 6.5. There is a grading on $H$ compatible with the isomorphism of Proposition 6.4 (2). Namely, if $\sigma \in G$ then the vector space $H_{\sigma}$ has basis $\left\{x_{1}^{s_{1}} \ldots x_{\theta}^{s_{\theta}} \sigma\right\}$. Define $H^{(i)}=\oplus_{j=0}^{m-1} H_{\chi^{m j+i}}$, thus $H=\oplus_{j=0}^{m-1} H^{(j)}$. It is not difficult to prove that with this grading $H$ is a $C_{m}$-crossed product (see definition 5.7) and this crossed product is compatible with the isomorphism of Proposition 6.4 (2).

6.3. Right simple $A(H, s)$-comodule algebras. We shall present some families of right $A(H, s)$-simple left $A(H, s)$-comodule algebras. This class will be big enough to classify module categories over $\operatorname{Rep}(A(H, s))$ in some cases.

Let $(K, \lambda)$ be a finite-dimensional left $H$-comodule algebra. We say that $(K, \lambda)$ is of type 1 if the following assumptions are satisfied:

- There exists a subgroup $F \subseteq C_{m^{2}}$ and $t \in \mathbb{N}$ such that $K$ has a basis $\left\{y_{1}^{r_{1}} \ldots y_{t}^{r_{t}} e_{f}: 0 \leq r_{j}<N_{j}, f \in F, t \leq \theta\right\}$ such that

$$
e_{\chi^{a}} y_{l}=q^{a d_{l}} y_{l} e_{\chi^{a}}, \quad \text { if } \chi^{a} \in F \text {. }
$$


- there is an inclusion $\iota: K \hookrightarrow H$ of $H$-comodules such that

$$
\iota\left(e_{f}\right)=f, \quad \iota\left(y_{l}\right)=x_{l},
$$

for all $f \in F, l=1 \ldots t$.

Observe that in this case we have that

$$
\lambda\left(e_{f}\right)=f \otimes e_{f}, \quad \lambda\left(y_{l}\right)=x_{l} \otimes 1+\chi^{-b_{l}} \otimes y_{l} .
$$

Definition 6.6. We shall say that a Hopf algebra $H=\mathfrak{B}(V) \# \mathbb{k} G$ is of type 1 if

(1) $\mathfrak{B}(V)$ has a basis $\left\{x_{1}^{s_{1}} \ldots x_{\theta}^{s_{\theta}}: 0 \leq s_{i} \leq N_{i}\right\}$, where $V$ is the vector space generated by $\left\{x_{1}, \ldots, x_{\theta}\right\}$,

(2) any right $H$-simple left $H$-comodule algebra $(K, \lambda)$ is equivariantly Morita equivalent to a comodule algebra of type 1 .

Remark 6.7. If $H=\mathfrak{B}(V) \# \mathbb{k} \Gamma$ is the bosonization of a Nichols algebra and a group algebra a finite group $\Gamma$ then $H$ is of type 1 when $V$ is a quantum linear space and $\Gamma$ is an Abelian group [Mo2] or when $V$ is constructed from a rack and $\Gamma=\mathbb{S}_{3}, \mathbb{S}_{4}[\mathrm{GM}]$.

Let $(K, \lambda)$ be a type 1 left $H$-comodule algebra such that $K_{0}=\mathbb{k} F$ where $F \subseteq C_{m^{2}}$ is a subgroup such that $\langle\sigma\rangle \subseteq F$ we shall denote by $\lambda^{J_{s}}: K \rightarrow H \otimes K$ the map given by

$$
\lambda^{J_{s}}(x)=J_{s} \lambda(x) J_{s}^{-1}, \quad \text { for all } x \in K .
$$

Here $J_{s}$ is identified with an element in $H \otimes K$ via the inclusion id ${ }_{H} \otimes \iota$. The same calculation as in [A. Prop. 3.1.1] proves that $\lambda^{J_{s}}(K) \subseteq H \otimes K$. Define $\left(K^{J_{s}}, \lambda^{J_{s}}, \Phi_{s}\left(J_{s} \otimes 1\right)\right)$ the left $H$-comodule algebra with underlying algebra $K^{J_{s}}$, coaction $\lambda^{J_{s}}$ and associator $\Phi_{s}\left(J_{s} \otimes 1\right)$. It follows from Lema 4.9 that $\left(K^{J_{s}}, \lambda^{J_{s}}, \Phi_{s}\right)$ is a left $H_{J_{s}}$-comodule algebra.

Lemma 6.8. The left $H$-comodule algebras $(K, \lambda)$ and $\left(K^{J_{s}}, \lambda^{J_{s}}, \Phi_{s}\left(J_{s} \otimes 1\right)\right)$ are equivariantly Morita equivalent, that is ${ }_{K} \mathcal{M},{ }_{K}^{J_{s}} \mathcal{M}$ are equivalent as $\operatorname{Rep}(H)$-modules.

Proof. For any $X \in \operatorname{Rep}(H), M \in{ }_{K} \mathcal{M}$ and any $x \in X, m \in M$ define

$$
c_{X, M}: X \otimes_{\mathbb{k}} M \rightarrow X \otimes_{\mathbb{k}} M, \quad c_{X, M}(x \otimes m)=J_{s} \cdot(x \otimes m) .
$$

It is immediate to prove that the identity functor (Id, $c$ ) $:{ }_{K} \mathcal{M} \rightarrow{ }_{K^{J_{s}}} \mathcal{M}$ is an equivalence of module categories.

Definition 6.9. Let $\left(\mathcal{K}, \lambda, \Phi^{\lambda}\right)$ be a left $H_{J_{s}}$-comodule algebra such that the associator $\Phi^{\lambda} \in A(H, s) \otimes_{\mathbb{k}} A(H, s) \otimes_{\mathbb{K}} \mathcal{K}$. Define $\widehat{\mathcal{K}}=\lambda^{-1}\left(A(H, s) \otimes_{\mathbb{k}} \mathcal{K}\right)$ and denote $\widehat{\lambda}$ the restriction of $\lambda$ to $\widehat{\mathcal{K}}$. Then $\left(\widehat{\mathcal{K}}, \widehat{\lambda}, \Phi_{s}\right)$ is a left $A(H, s)$-comodule algebra. Turns out that this procedure is the inverse of the crossed product. 
6.4. Actions on module categories ${ }_{\left(\widehat{K}, \widehat{\lambda}, \Phi_{s}\right)} \mathcal{M}$. For the rest of this section we shall assume now that $m=p$ is a prime number.

Let $(K, \lambda)$ be a type 1 left $H$-comodule algebra such that $K_{0}=\mathbb{k} F$ where $F=C_{d}$ is a cyclic group.

There are two possible cases; when $\langle\sigma\rangle \subseteq F$ or $F=\{1\}$. Let us treat the first case. So we assume that $p \mid d$. Let $s, l \in \mathbb{N}$ be such that $d=p s$ and $s l=p$. Let us denote $\widehat{F}=C_{s}=<\chi^{l p}>$.

By hypothesis the vector space $K$ has a decomposition $K=\oplus_{f \in F} K_{f}$ where $K_{f}$ is the vector space with basis $\left\{y_{1}^{r_{1}} \ldots y_{t}^{r_{t}} e_{f}: 0 \leq r_{j} \leq N_{j}\right\}$. For any $i=0 \ldots s-1$ define

$$
K^{(i)}=\bigoplus_{j: \chi^{m j+i} \in C_{d}} K_{\chi^{m j+i}}
$$

Observe that $\widehat{K}=K^{(0)}$. With this grading $K$ is an $\widehat{F}$-crossed product.

Lemma 6.10. Under the above assumptions ${ }_{\left(\widehat{K}, \widehat{\lambda}, \Phi_{s}\right)} \mathcal{M}$ is an $\widehat{F}$-equivariant $\operatorname{Rep}(A(H, s))$-module category and $\left({ }_{\left(\widehat{K}, \widehat{\lambda}, \Phi_{s}\right)} \mathcal{M}\right)^{\widehat{F}} \simeq{ }_{K} \mathcal{M}$ as module categories over $\operatorname{Rep}(H)$.

Proof. It follows from Proposition 5.13 and Proposition 5.14 .

Now, let us assume that $F=\{1\}$. Let us endow the space $K \otimes_{\mathbb{k}} \mathbb{k} C_{p}$ with the product determined by

$$
\left(y_{l} \otimes \sigma^{a}\right)\left(y_{s} \otimes \sigma^{b}\right)=q^{p a d_{s}} y_{l} y_{s} \otimes \sigma^{a+b} .
$$

The space $K \otimes_{\mathbb{k}} \mathbb{k} C_{p}$ is a left $H$-comodule algebra with coproduct determined by

$$
\lambda\left(y_{l} \otimes \sigma^{a}\right)=x_{l} \sigma^{a} \otimes 1 \otimes \sigma^{a}+\sigma^{a} \chi^{-b_{l}} \otimes y_{l} \otimes \sigma^{a} .
$$

It is clear that $\left(K \otimes_{\mathbb{k}} \mathbb{k} C_{p}\right)_{0}=\mathbb{k} C_{p}$. Thus we can consider the left $A(H, s)$ comodule algebra $\left(K \otimes_{\mathbb{k}} \mathbb{k} C_{p}, \widehat{\lambda}, \Phi_{s}\right)$.

Lemma 6.11. Under the above conventions the following holds.

1. The module category $\left(\mathbb{k}_{\left.p_{p}, \lambda, \Phi_{s}\right)} \mathcal{M}\right.$ has a $C_{p}$-action such that there $i s$ an equivalence $\left({ }_{\left(\mathbb{k} C_{p}, \lambda, \Phi_{s}\right)} \mathcal{M}\right)^{C_{p}} \simeq \operatorname{Vect}_{\mathbb{k}}$ as $\operatorname{Rep}(H)$-modules.

2. The module category ${ }_{\left(K \otimes_{\mathbb{k}} \mathbb{k}_{p}, \widehat{\lambda}, \Phi_{s}\right)} \mathcal{M}$ has a $C_{p}$-action such that there is an equivalence $\left({ }_{\left(K \otimes_{\mathbb{k}} \mathbb{k} C_{p}, \widehat{\lambda}, \Phi_{s}\right)} \mathcal{M}\right)^{C_{p}} \simeq{ }_{K} \mathcal{M}$ as $\operatorname{Rep}(H)$-modules.

Proof. 1. It follows from (2) taking $K=\mathbb{k}$.

2. Set $\mathcal{M}={ }_{\left(K \otimes_{\mathbb{k}} \mathbb{k} C_{p}, \widehat{\lambda}, \Phi_{s}\right)} \mathcal{M}$. For any $i=0, \ldots, p-1$ define the functors $\left(U_{i}, c^{i}\right): \mathcal{M} \rightarrow \mathcal{M}^{\sigma^{i}}$ as follows. For any $M \in \mathcal{M} U_{i}(M)=M$ with a new action $\triangleright:\left(K \otimes_{\mathbb{k}} \mathbb{k} C_{p}\right) \otimes_{\mathbb{k}} M \rightarrow M$ of $K \otimes_{\mathbb{k}} \mathbb{k} C_{p}$ given by

$$
y_{l} \triangleright m=q^{i d_{l}} y_{l} \cdot m, \quad \sigma \triangleright m=q^{i p} \sigma \cdot m,
$$


for all $l=1, \ldots, t, m \in M$. For any $X \in \operatorname{Rep}(A), M \in \mathcal{M}$ the map $c_{X, M}^{i}: U_{i}\left(X \otimes_{\mathbb{k}} M\right) \rightarrow F_{i}(X) \otimes_{\mathbb{k}} U_{i}(M)$ is the identity.

The isomorphism $\mu_{i, j}: U_{i} \circ U_{j} \rightarrow U_{i+j}$ is given by the action of $\sigma^{-\frac{(i+j)-(i+j)^{\prime}}{p}}$. Altogether makes the category ${ }_{\left(K \otimes_{\mathbb{k}} \mathbb{k} C_{p}, \widehat{\lambda}, \Phi_{s}\right)} \mathcal{M}$ a $C_{p}$-equivariant $\operatorname{Rep}(A)$ module category.

Let $N \in{ }_{K} \operatorname{Mod}$. Define $\mathcal{F}(N)=\oplus_{i=0}^{p-1} N_{i}$ where $N_{i}=N$ as vector spaces. Let us define a new action of $\rightarrow: K \otimes_{\mathbb{k}} \mathbb{k} C_{p} \otimes_{\mathbb{k}} \mathcal{F}(N) \rightarrow \mathcal{F}(N)$ as follows. If $n \in N_{i}$ then

$$
\sigma \rightarrow n=q^{p i} n \in N_{i}, \quad y_{l} \rightarrow n=q^{d_{l} i} y_{l} \cdot n \in N_{\left(d_{l}+i\right)^{\prime}} .
$$

Recall that $a^{\prime}$ denotes the remainder of $a$ in the division by $p$. Note also that for any $i, j=0, \ldots, p-1 U_{i}\left(N_{j}\right)=N_{i+j}$. The module $\mathcal{F}(N)$ is a $C_{p^{-}}$

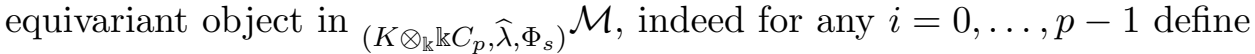
the isomorphisms $v_{i}: U_{i}(\mathcal{F}(N)) \rightarrow \mathcal{F}(N)$ as follows: $v_{i}(n)=q^{-i} n \in N_{i+j}$ for any $n \in N_{j}$. This maps are $K \otimes_{\mathbb{k}} \mathbb{k} C_{p}$-module isomorphisms and they satisfy equation (3.4). This defines a functor $\mathcal{F}:{ }_{K} \operatorname{Mod} \rightarrow{ }_{\left(K \otimes_{\mathbb{k}} \mathbb{k} C_{p}, \widehat{\lambda}, \Phi_{s}\right)} \mathcal{M}$ that together with the identity isomorphisms $c_{X, N}: \mathcal{F}\left(X \otimes_{\mathbb{k}} N\right) \rightarrow X \otimes_{\mathbb{k}} \mathcal{F}(N)$ becomes a module functor.

If $M \in{ }_{\left(K \otimes_{\mathbb{k}} \mathbb{k} C_{p}, \widehat{\lambda}, \Phi_{s}\right)} \mathcal{M}$ then $M=\oplus_{i=0}^{p-1} M_{i}$ where $M_{i}$ is the eigenspace of the eigenvalue $q^{p i}$ of the action of $\sigma$. The space $M_{0}$ has a $K$-action as follows. Since $M$ is $C_{p}$-equivariant there are isomorphisms $v_{i}: U_{i}(M) \rightarrow M$ such that the restrictions $\left.v_{i}\right|_{M_{0}}: M_{0} \rightarrow M_{i}$ are isomorphisms. If $m \in M_{0}$, $y_{l} \in K$ then $y_{l} \cdot m \in M_{d_{l}}$, thus we can define $\rightarrow: K \otimes_{\mathbb{k}} M_{0} \rightarrow M_{0}$

$$
y_{l} \rightarrow m=v_{d_{l}}^{-1}\left(y_{l} \cdot m\right),
$$

for all $m \in M_{0}$. The map $M \mapsto M_{0}$ is functorial and defines an inverse functor for $\mathcal{F}$.

6.5. Exact module categories over $\operatorname{Rep}(A(H, s))$. Now we can formulate the main result of this section.

Theorem 6.12. Let $H$ be a Hopf algebra of type 1 (see definition [6.6) and let $\mathcal{M}$ be an exact indecomposable module category over $\operatorname{Rep}(A(H, s))$. Then the following statements hold.

(1) there exists a right $H$-simple left $H$-comodule algebra $(K, \lambda)$ with trivial coinvariants such that $K_{0} \supseteq C_{p}$ and there is an equivalence of module categories $\mathcal{M} \simeq{ }_{\left(\widehat{K}, \widehat{\lambda}, \Phi_{s}\right)} \mathcal{M}$.

(2) If there is an equivalence ${ }_{\left(\widehat{K^{\prime}}, \widehat{\lambda}^{\prime}, \Phi_{s}^{\prime}\right)} \mathcal{M} \simeq{ }_{\left(\widehat{K}, \widehat{\lambda}, \Phi_{s}\right)} \mathcal{M}$ as $\operatorname{Rep}(A(H, s))$ modules then $(K, \lambda)$ and $\left(K^{\prime}, \lambda^{\prime}\right)$ are equivariantly Morita equivalent $H$-comodule algebras.

Proof. 1. By Lemma 4.7 there exists a left $A(H, s)$-comodule algebra $(\mathcal{K}, \lambda, \Phi)$ such that $\mathcal{M} \simeq \mathcal{K} \mathcal{M}$. The category $\mathcal{K} \mathcal{M}$ is $F$-equivariant for some subgroup $F \subseteq C_{p}$. Thus it follows from [AM, Thm 3.3] that there is a right 
$H$-simple left $H$-comodule algebra $(S, \delta)$ with trivial coinvariants such that $(\mathcal{K} \mathcal{M})^{F} \simeq{ }_{S} \mathcal{M}$ as $\operatorname{Rep}(H)$-modules. Hence $S_{0}=\mathbb{k} 1, S_{0}=\mathbb{k} C_{p}$ or $S_{0}=\mathbb{k} C_{p^{2}}$. In any case, it follows from Lemmas 6.10, 6.11 that there is a right $H$ simple left $H$-comodule algebra $(K, \lambda)$ with trivial coinvariants such that $K_{0} \supseteq C_{p}$ and there is an equivalence ${ }_{S} \mathcal{M} \simeq\left({\left.\widehat{\left(K^{\prime}\right.},{\widehat{\lambda^{\prime}}}^{\prime}, \Phi_{s}^{\prime}\right)}^{\mathcal{M}}\right)^{F}$. Whence $(\mathcal{K} \mathcal{M})^{F} \simeq\left({\left.\widehat{\left(K^{\prime}\right.}, \widehat{\lambda^{\prime}}, \Phi_{s}^{\prime}\right)}^{\mathcal{M}}\right)^{F}$, thus using Proposition 3.4 (5) we get the result.

2. There exists a subgroup $F \subseteq C_{p}$ such that both module categories $\left(\widehat{K^{\prime}}, \widehat{\lambda}^{\prime}, \Phi_{s}^{\prime}\right) \mathcal{M},{ }_{\left(\widehat{K}, \widehat{\lambda}, \Phi_{s}\right)} \mathcal{M}$ are $F$-equivariant and there are equivalences of module categories over $\operatorname{Rep}\left(H_{J_{s}}\right)$

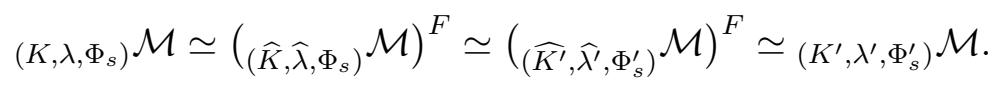

Thus by Lemma 6.8 follows that ${ }_{K} \mathcal{M} \simeq{ }_{K^{\prime}} \mathcal{M}$.

6.6. Some classification results. We apply Theorem 6.12 to obtain the classification of module categories over $\operatorname{Rep}(A(H, s))$ where $H$ is the bosonization of a quantum linear space.

Let $g_{1}, \ldots, g_{\theta} \in C_{p^{2}}, \chi_{1}, \ldots, \chi_{\theta} \in \widehat{C_{p^{2}}}$ be a datum for a quantum linear space and let $V=V\left(g_{1}, \ldots, g_{\theta}, \chi_{1}, \ldots, \chi_{\theta}\right)$ the associated Yetter-Drinfeld module over $\mathbb{k} C_{p^{2}}$ generated as a vector space by $x_{1}, \ldots, x_{\theta}$. For more details see $\mathrm{AS}]$.

The Hopf algebra $H=\mathfrak{B}(V) \# \mathbb{k} C_{p^{2}}$ is a type 1 Hopf algebra, see [Mo2].

Let us define now a family of right $H$-simple left $H$-comodule algebras. Let $F \subseteq C_{p^{2}}$ be a subgroup and $\xi=\left(\xi_{i}\right)_{i=1 \ldots \theta}, \alpha=\left(\alpha_{i j}\right)_{1 \leq i<j \leq \theta}$ be two families of elements in $\mathbb{k}$ satisfying

$$
\begin{gathered}
\xi_{i}=0 \text { if } g_{i}^{N_{i}} \notin F \text { or } \chi_{i}^{N_{i}}(f) \neq 1, \\
\alpha_{i j}=0 \text { if } g_{i} g_{j} \notin F \text { or } \chi_{i} \chi_{j}(f) \neq 1,
\end{gathered}
$$

for all $f \in F$. In this case we shall say that the pair $(\xi, \alpha)$ is a compatible comodule algebra datum with respect to the quantum linear space $V$ and the group $F$.

The algebra $\mathcal{A}(V, F, \xi, \alpha)$ is the algebra generated by elements in $\left\{v_{i}: i=\right.$ $1 \ldots \theta\},\left\{e_{f}: f \in F\right\}$ subject to relations

$$
\begin{gathered}
e_{f} e_{g}=e_{f g}, \quad e_{f} v_{i}=\chi_{i}(f) v_{i} e_{f}, \\
v_{i} v_{j}-q_{i j} v_{j} v_{i}= \begin{cases}\alpha_{i j} e_{g_{i} g_{j}} & \text { if } g_{i} g_{j} \in F \\
0 & \text { otherwise }\end{cases} \\
v_{i}^{N_{i}}= \begin{cases}\xi_{i} e_{g_{i}^{N_{i}}} & \text { if } g_{i}^{N_{i}} \in F \\
0 & \text { otherwise }\end{cases}
\end{gathered}
$$


for any $1 \leq i<j \leq \theta$. If $W \subseteq V$ is a $\mathbb{k} C_{p^{2}}$-subcomodule invariant under the action of $F$, we define $\mathcal{A}(W, F, \xi, \alpha)$ as the subalgebra of $\mathcal{A}(V, F, \xi, \alpha)$ generated by $W$ and $\left\{e_{f}: f \in F\right\}$.

The algebras $\mathcal{A}(V, F, \xi, \alpha)$ are right $H$-simple left $H$-comodule algebras with coaction determined by

$$
\lambda\left(v_{i}\right)=x_{i} \otimes 1+g_{i} \otimes v_{i}, \quad \lambda\left(e_{f}\right)=f \otimes e_{f},
$$

for all $i=1, \ldots, \theta, f \in F$. The subalgebras $\mathcal{A}(W, F, \xi, \alpha)$ are also right $H$-simple left $H$-subcomodule algebras.

Theorem 6.13. [Mo2, Thm 4.6, Thm. 4.9] Let $\mathcal{M}$ be an exact indecomposable module category over $\operatorname{Rep}(H)$.

1. There exists a subgroup $F \subseteq C_{p^{2}}$, a compatible datum $(\xi, \alpha)$ and $W \subseteq V$ a subcomodule invariant under the action of $F$ such that $\mathcal{M} \simeq \mathcal{A}(W, F, \xi, \alpha) \mathcal{M}$ as module categories.

2. The left $H$-comodule algebras $\mathcal{A}(W, F, \xi, \alpha), \mathcal{A}\left(W^{\prime}, F^{\prime}, \xi^{\prime}, \alpha^{\prime}\right)$ are equivariantly Morita equivalent if and only if $(W, F, \xi, \alpha)=\left(W^{\prime}, F^{\prime}, \xi^{\prime}, \alpha^{\prime}\right)$.

Given a compatible datum $(\xi, \alpha)$ with respect to $V$ and $C_{p}$ define the left $A(H, s)$-comodule algebra $\widehat{\mathcal{A}}(V, \xi, \alpha)$ with underlying algebra equal to $\mathcal{A}\left(V, C_{p}, \xi, \alpha\right)$ and coaction $\widehat{\lambda}: \widehat{\mathcal{A}}(V, \xi, \alpha) \rightarrow A(H, s) \otimes_{\mathbb{k}} \widehat{\mathcal{A}}(V, \xi, \alpha)$ given by $\widehat{\lambda}(a)=J_{s} \lambda(a) J_{s}^{-1}$ for all $a \in \widehat{\mathcal{A}}(V, \xi, \alpha)$. If $W \subseteq V$ is a $\mathbb{k} C_{p^{2}}$-subcomodule invariant under the action of $C_{p}$ define $\widehat{\mathcal{A}}(W, \xi, \alpha)$ as the subalgebra of $\widehat{\mathcal{A}}(V, \xi, \alpha)$ generated by $W$ and $C_{p}$.

As a consequence of Theorem 6.12 we have the following result.

Theorem 6.14. Let $\mathcal{M}$ be an exact indecomposable module category over $\operatorname{Rep}(A(H, s))$.

1. There exists a compatible datum $(\xi, \alpha)$ and $W \subseteq V$ a subcomodule invariant under the action of $C_{p}$ such that there is an equivalence $\mathcal{M} \simeq \widehat{\widehat{\mathcal{A}}(W, \xi, \alpha)}, \mathcal{M}$ as $\operatorname{Rep}(A(H, s))$-module categories.

2. The comodule algebras $\widehat{\mathcal{A}}(W, \xi, \alpha), \widehat{\mathcal{A}}\left(W^{\prime}, \xi^{\prime}, \alpha^{\prime}\right)$ are equivariantly Morita equivalent if and only if $(W, \xi, \alpha)=\left(W^{\prime}, \xi^{\prime}, \alpha^{\prime}\right)$.

\section{REFERENCES}

[A] I. Angiono. Basic quasi-Hopf algebras over cyclic groups. Adv. Math. 225 (2010), 3545-3575.

[AM] N. Andruskiewitsch and M. Mombelli. On module categories over finitedimensional Hopf algebras. J. Algebra 314 (2007), 383-418.

[AS] N. ANDRUsKiEwitsch and H.-J. SCHNEIDER. Lifting of quantum linear spaces and pointed Hopf algebras of order $p^{3}$. J. Algebra 209 (1998), 658-691.

[BEK] J. Böckenhauer, D. E. Evans and Y. Kawahigashi. Chiral Structure of Modular invariants for Subfactors. Commun. Math. Phys. 210 (2000), 733-784. 
[BFS] T. Barmeier, J. Fuchs and C. Schweigert. Module categories for permutation modular invariants. Int. Math. Res. Not. 16 (2010) 3067-310.

[BO] R. BezRukavnikov and V. Ostrik. On tensor categories attached to cells in affine Weyl groups II. Advanced Studies in Pure Mathematics 40 (2004), 101119.

[CS1] R. Coquereaux and G. Schieber. Orders and dimensions for $\mathfrak{s l}_{2}$ and $\mathfrak{s l}_{3}$ module categories and boundary conformal field theories on a torus. J. Math. Phys. 48, 043511 (2007).

[CS2] R. Coquereaux and G. Schieber. From conformal embeddings to quantum symmetries: an exceptional SU(4) example. Journal of Physics- Conference Series Volume 103 (2008), 012006.

[D] V. Drinfeld. Quasi-Hopf algebras. (Russian) Algebra i Analiz 1 (1989), no. 6, 114-148; translation in Leningrad Math. J. 1 (1990), no. 6, 1419-1457.

[DGNO] V. Drinfeld, S. Gelaki, D. Nikshych and V. Ostrik. On Braided Fusion Categories I. Selecta Math. N.S. 16, 1 (2010) 1-119.

[EG1] P. Etingof and S. Gelaki. Finite-dimensional quasi-Hopf algebras with radical of codimension 2. Math. Res. Lett. 11 (2004), 685-696.

[EG2] P. Etingof and S. Gelaki. On radically graded finite-dimensional quasi-Hopf algebras. Mosc. Math. J. 5 (2005), no. 2, 371-378.

[EG3] P. ETINGOF and S. Gelaki. Liftings of graded quasi-Hopf algebras with radical of prime codimension. J. Pure Appl. Algebra 205, No.2, 310-322 (2006).

[EN] P. Etingof and D. Nikshych. Dynamical twists in group algebras. Int. Math. Res. Not. 13 (2001), 679-701.

[ENO1] P. Etingof, D. Nikshych and V. Ostrik. On fusion categories. Ann. Math. 162, 581-642 (2005).

[ENO2] P. Etingof, D. Nikshych and V. Ostrik. Weakly group-theoretical and solvable fusion categories. Adv. Math 226, 15 (2011), 176-205.

[ENO3] P. Etingof, D. Nikshych and V. Ostrik. Fusion categories and homotopy theory. Quantum Topol. 1, No. 3, (2010) 209-273.

[EO1] P. Etingof and V. Ostrik. Finite tensor categories. Mosc. Math. J. 4 (2004), no. 3, 627-654.

[EO2] P. Etingof and V. OstRik. Module categories over representations of $S L_{q}(2)$ and graphs. Math. Res. Lett. (1) 11 (2004) 103-114.

[FS1] J. Fuchs and C. SchweigerT. Category theory for conformal boundary conditions. Vertex Operator Algebras in Mathematics and Physics, Fields Institute Comm. 39 (2003) 25-70.

[FS2] J. Fuchs and C. SCHWEIGERT. Hopf algebras and finite tensor categories in conformal field theory. Rev. Unión Mat. Argent. (2) 51 (2010) 43-90.

[Ga1] C. Galindo. Clifford theory for tensor categories. J. Lond. Math. Soc. (2) 83 (2011) 57-78.

[Ga2] C. Galindo. Clifford theory for graded fusion categories. Israel J. Math. to appear. Preprint arXiv: 1010.5283 .

[Ge] S. Gelaki. Basic quasi-Hopf algebras of dimension $n^{3}$. J. Pure Appl. Algebra 198, No. 1-3, (2005) 165-174.

[GM] A. García Iglesias and M. Mombelli. Representations of the category of modules over pointed Hopf algebras over $\mathbb{S}_{3}$ and $\mathbb{S}_{4}$. Pac. J. Math to appear. Preprint arxiv: 1006.1857.

[Gi] V. GinzBurg. Calabi-Yau Algebras. Preprint arxiv:math/0612139.

[Gr] J. Greenough. Monoidal 2-structure of Bimodule Categories. J. Algebra 324 (2010) 1818-1859.

[GS] P. Grossman and N. SNyder. Quantum subgroups of the Haagerup fusion categories. Preprint arXiv: 1102.2631. 
[He] H. HENKER. Freeness of quasi-Hopf algebras over right coideal subalgebras. Commun. Algebra 38 (2010) 876-889.

[KO] A. KIRILlov JR. and V. Ostrik. On a q-analogue of the McKay correspondence and the $A D E$ classification of $s_{2}$ conformal field theories. Adv. Math. 171 (2002), no. 2, 183-227.

[MM] E. MeIr, E. Musicantov. Module categories over graded fusion categories. Preprint arxiv: 1010.4333.

[Mo1] M. Mombelli, Module categories over pointed Hopf algebras. Math. Z. 266 (2010) 319-344.

[Mo2] M. MombelLi. Representations of tensor categories coming from quantum linear spaces. J. Lond. Math. Soc. (2) 83 (2011) 19-35.

[Na] S. NATALE. Hopf algebra extensions of group algebras and Tambara-Yamagami categories. Algebr. Represent. Theory 13 (6), (2010) 673-691.

[N] D. Nikshych. Non group-theoretical semisimple Hopf algebras from group actions on fusion categories. Selecta Math. 14 (2008), 145-161.

[Oc] A. OCNEANU. The classification of subgroups of quantum $S U(N)$, in Quantum symmetries in theoretical physics and mathematics (Bariloche, 2000). Contemp. Math. 294 (2002), 133-159.

[O1] V. Ostrik. Module categories, Weak Hopf Algebras and Modular invariants. Transform. Groups, 2 8, 177-206 (2003).

[O2] V. Ostrik. Module categories over the Drinfeld double of a Finite Group. Int. Math. Res. Not. 2003, no. 27, 1507-1520.

[O3] V. Ostrik. Module Categories Over Representations of $S L_{q}(2)$ in the NonSemisimple Case. Geom. Funct. Anal. Vol. 17 (2008), 2005-2017.

[Ta] D. TAMBARA. Invariants and semi-direct products for finite group actions on tensor categories. J. Math. Soc. Japan 53 (2001), 429-456.

Departamento de Matemáticas, Universidad de los Andes,

Carrera $1 \mathrm{~N}^{\circ}$ 18A - 12 Bogotá, Colombia

E-mail address: cn.galindo1116@uniandes.edu.co, cesarneyit@gmail.com

URL: http://matematicas.uniandes.edu.co/ cgalindo/

Facultad de Matemática, Astronomía y Física,

Universidad NACIONAL DE CóRdoba,

Medina Allende s/n, (5000) Ciudad Universitaria,

Córdoba, Argentina

E-mail address: martin10090@gmail.com, mombelli@mate.uncor.edu

URL: http://www.mate.uncor.edu/ mombelli 